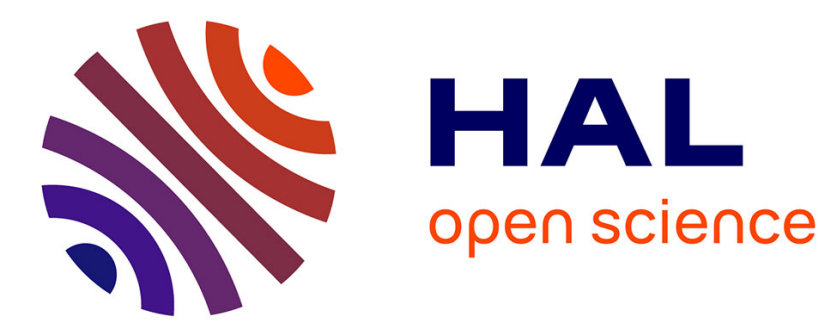

\title{
Fault Tolerant MPC Design for Reliable Microgrid Energy Management under Uncertainties
}

Ionela Prodan, Enrico Zio, Florin Stoican

\section{To cite this version:}

Ionela Prodan, Enrico Zio, Florin Stoican. Fault Tolerant MPC Design for Reliable Microgrid Energy Management under Uncertainties. Energy, 2015, 91, pp.20-34. 10.1016/j.energy.2015.08.009 . hal01259767

\section{HAL Id: hal-01259767 \\ https://hal.science/hal-01259767}

Submitted on 20 Jan 2016

HAL is a multi-disciplinary open access archive for the deposit and dissemination of scientific research documents, whether they are published or not. The documents may come from teaching and research institutions in France or abroad, or from public or private research centers.
L'archive ouverte pluridisciplinaire HAL, est destinée au dépôt et à la diffusion de documents scientifiques de niveau recherche, publiés ou non, émanant des établissements d'enseignement et de recherche français ou étrangers, des laboratoires publics ou privés. 


\title{
Fault Tolerant MPC Design for Reliable Microgrid Energy Management under Uncertainties
}

\author{
Ionela Prodan ${ }^{\mathrm{a}}$, Enrico Zio ${ }^{\mathrm{b}}$, Florin Stoican ${ }^{\mathrm{c}}$ \\ ${ }^{a}$ Laboratory of Conception and Integration of Systems (LCIS EA 3747), Univ. Grenoble Alpes, \\ F-26902, France, ionela.prodan@lcis.grenoble-inp.fr \\ ${ }^{b}$ Chair on Systems Science and the Energetic Challenge, European Foundation for New Energy - \\ EDF, CentraleSupélec, Gif sur Yvette 91192, France, Department of Energy, Politecnico di \\ Milano, Milano, Italy, enrico.zio@centralesupelec.fr \\ ${ }^{c}$ Department of Automatic Control and Systems Engineering, UPB, Bucharest, Romania, \\ florin.stoican@acse.pub.ro
}

\begin{abstract}
This paper presents an extension of a Model Predictive Control (MPC) approach for microgrid energy management which takes into account electricity costs, power consumption, generation profiles, power and energy constraints as well as uncertainty due to variations in the environment. The approach is based on a coherent framework of control tools, like mixed-integer programming and soft constrained MPC, for describing the microgrid components dynamics and the overall system control architecture. Fault tolerant strategies are inserted in order to ensure the proper amount of energy in the storage devices such that (together with the utility grid) the essential consumer demand is always covered. Simulation results on a particular microgrid architecture validate the proposed approach.
\end{abstract}

Keywords: Microgrid energy management, Uncertainty, Model Predictive Control (MPC), Mixed-Integer Linear Programming (MILP), Fault Tolerant Control (FTC), Soft constraints.

\section{Introduction}

Microgrids are increasingly considered for system solutions including distributed energy resources Liang and Zhuang (2014), Lund (2007), Foley et al. (2010), Johansson (2013). This is also because they can help both prepare for, and prevent the threat of climate change. Combating climate change necessarily involves a critical shift away from fossil fuels and towards clean energy, efficiency, and renewable en- 
ergy. For this, energy resources are inherently distributed and resilient, which makes them naturally compatible with a microgrid system solution.

The flexible configuration and operation of microgrids helps avoid cascading failures and, thus, blackouts and losses of stability. Microgrids can be attached to the utility (grid-connected) and isolated (islanded) easily in case of faults or instability in the external grid. Once the disturbance in the main grid has cleared, microgrids can be connected again and system reliability is improved Lo Prete et al. (2012), Bracco et al. (2014).

Realistic modeling and optimization for efficient, reliable and economic planning, operation and control of microgrids are very important and still open issues. Various techniques for the minimization of microgrid operating costs include robust optimization Rahimiyan et al. (2014), heuristics Sanseverino et al. (2011), mathematical programming Hawkes et al. (2009) and priority rules Tsikalakis and Hatziargyriou (2011). Only recently Model Predictive Control (MPC) has started to have a growing interest between researcher in energy filed, in particular in microgrids applications (see, for instance Rawlings and Mayne (2009) for detailed notions on MPC). A method based on the combination of empirical mean, dynamic programming and MPC has been used in Hooshmand et al. (2012) for solving a power management problem within a microgrid in islanded mode operation. In Parisio and Glielmo (2011), a preliminary study has addressed the application of a receding horizon approach for optimizing microgrid operations while satisfying time-varying demands and operational constraints. In Pérez et al. (2013), an approach based on MPC has been designed to manage in real-time the power production of a grid-tied photovoltaic plus energy storage power plant with a reduced energy storage system capacity. It is important to mention that all these papers do not consider explicitly the detailed modeling of the microgrid components, the constraints description and the interaction between the independent components of the micrigrid system. Instead, abstract mathematical models are used to embody the practical and functional behavior of the components and the focus is on the formulation of the optimization problem for minimizing costs. In Negenborn et al. (2009) a model predictive controller is applied for controlling the energy flows inside a household system equipped with a "micro" combined heat and power unit. In addition, the household can buy and sell electricity from/to the energy supplier; heat and electricity can be stored in specific storage devices. In Zervas et al. (2008), MPC is used for energy scheduling on a hydrogen-based microgrid without batteries. In Qi et al. (2012), predictive control is applied for renewable energy power management with battery storage in a water desalination plant. In Khalid and Savkin (2010), MPC is performed for a plug-in renewable energy source with battery storage. The electrical power transferred to 
the network and that stored in the battery are the control variables considered. Finally, del Real et al. (2007) presents the application of hybrid modeling control for a photovoltaic-fuel cell power plant.

In this paper a microgrid is considered for exemplification, connected to the utility grid via a distribution transformer and containing local consumers, renewable generators (wind turbines or photovoltaic panels) and electrical storage facilities (denoted as batteries). These latter represent the key control components in the microgrid energy management. The overall objective of the microgrid system is to generate suitable decisions for all the source and electrical storage components in such a way to fulfill load demands. Figure 1 illustrates a typical smart energy management system. Based on the output of the forecasting unit, the optimization unit computes a control action such that an operation cost is minimized. Once an optimal controller is obtained, then it is sent to all the storages and sources which need to be controlled. We must precise that forecasting is out of the scope of the present paper, rather we consider real numerical data for the reference profiles as given in (Grigg et al., 1999).

[Figure 1 about here.]

The overall goal of this paper is to implement a realistic and flexible control scheme where:

- costs, constraints, profiles are taken into account into a centralized constrained optimization problem (i.e., via a model predictive control design);

- depending on external events and energy costs the user may receive only its essential demand or up to the entire desired demand;

- faults at the generator output level are explicitly accounted in both robust (by managing the minimal storage requirements) and adaptive fashion (change of constraints and costs, as a function of system state - healthy, under fault, under recovery).

In particular, the present work extends the optimization-based control approach developed in Prodan and Zio (2014a,b). More specifically, the original contributions are the following:

- A more realistic and complex benchmark problem replaces the one presented in Prodan and Zio (2014a,b). That is, we consider more realistic dynamics for the components of the microgrid (especially the key storage component, i.e., the battery) and also we consider that the operative profiles can be affected by noise and perturbations, thus requiring a robust control design. 
- The battery charge/discharge cycles are penalized in the cost function, in order to account for the battery wear and tear.

- The user load is partitioned into two components:

- essential loads, that is, demands of power related to essential processes (e.g., electricity in an operating room, fail-safe modules in critical systems) and that one must always try to meet;

- non-essential loads, that can be reduced or shed during supply constraints or emergency situations (e.g., standby devices, day-time lighting and the like).

- Reliable functioning of the microgrid is maintained under parameter variations, noises and fault events (e.g., generator output outages). Especially for the latter case, we provide an adaptive control which can handle fault events via subsequent control reconfiguration.

- Extensive simulation results are provided through different scenarios which validate the proposed fault tolerant predictive control scheme.

The microgrid energy control in this paper is done via a centralized scheme which assumes global state, inputs and outputs which appear in the description of the dynamics, constraints and costs. We proceed by defining and illustrating in the rest of the paper these signals and associated matrices, as needed.

The paper is organized as follows. Section 2 describes in detail the considered microgrid system. Section 3 presents the optimization-based control problem for efficient energy management and the considered strategies for fault tolerant control and battery wear and tear. Simulation results are provided in Section 4 and conclusions are drawn in Section 5.

[Figure 2 about here.]

\section{Microgrid system description}

Any microgrid, regardless on the particular constructive details, will contain several types of components as illustrated in Figure 2: power generators (e.g., hydro, wind turbine, photovoltaic panels and the like) denoted as

$$
\left\{G_{i}\right\}_{i=1 \cdots N_{g}} \text {, where } N_{g} \text { represents the number of generators, }
$$


storage elements (e.g., batteries)

$$
\left\{S_{j}\right\}_{j}=1 \cdots N_{s} \text {, where } N_{s} \text { represents the number of electrical storages, }
$$

consumers (e.g., large cooling houses),

$$
\left\{D_{k}\right\}_{k=1, \cdots, N_{d}} \text {, where } N_{d} \text { represents the number of consumers, }
$$

internal interconnections between these elements and (if not in islanded mode) external connections to the utility power grid denoted as $E$.

With reference to Figure 2, let us define in Table 1 the generic interconnection signals which appear in a typical microgrid system.

[Table 1 about here.]

Formally, the microgrid system is topologically characterized by a directed graph, as illustrated in Figure 2. The nodes are the previously described components and the edges are the links between these components. For the sake of keeping compact the system equations, let us introduce the adjacency matrices of Table 2 to characterize the links between components, e.g., $M_{g s}(i, j)=1$ means that there exists a connection between the generator $G_{i}$ and the storage unit $S_{j}$ (i.e., the link $u_{g s}^{i j}$ ) and $M_{g s}(i, j)=0$ denotes the absence of the connection.

[Table 2 about here.]

For future use, let us also define the number of non-zero entries in an adjacency matrix $^{1}$ :

$$
\begin{aligned}
N_{g s} & =\sum_{M_{g s}(i, j) \neq 0} M_{g s}(i, j), & N_{g d} & =\sum_{M_{g d}(i, k) \neq 0} M_{g d}(i, k), \\
N_{g e} & =\sum_{M_{g e}(i) \neq 0} M_{g e}(i), & N_{s d} & =\sum_{M_{s d}(j, k) \neq 0} M_{s d}(j, k), \\
N_{s e} & =\sum_{M_{s e}(j) \neq 0} M_{s e}(j), & N_{e d} & =\sum_{M_{e d}(k) \neq 0} M_{e d}(k),
\end{aligned}
$$

for all $i=1, \ldots, N_{g}, j=1, \ldots, N_{s}, k=1, \ldots, N_{d}$.

\footnotetext{
${ }^{1}$ For example, $N_{g s} \in \mathbb{R}$ denotes the number of non-zero entries in $M_{g s}$. In other words, $N_{g s}$ represents the number of interconnections between the components $G_{i}$ and $S_{j}$ of the microgrid.
} 
In the following, we detail the elements introduced from the point of view of control optimization: dynamics governing the internal functioning, profiles to be followed, constraints and costs affecting the components. Note that, since each of the components of a given type behaves similarly, we describe as much as possible generic rules of functioning and discuss particularities if and when it is the case.

\subsection{Electrical storage units}

\section{Storage dynamics}

In the proposed microgrid setting of Figure 2, the components that we assume to be directly controllable are the storage units (through the rates of charge and discharge). To model the operation in the charge and discharge modes, we introduce the following Linear-Time Invariant (LTI) dynamics²:

$$
x_{j}(t+1)=\left(1-\sigma_{j}\right) x_{j}(t)+\sum_{M_{g s}(i, j) \neq 0} u_{g s}^{i j}(t)-\sum_{M_{s d}(i, j) \neq 0} u_{s d}^{j k}(t)-\sum_{M_{s e}(j, k) \neq 0} u_{s e}^{j}(t)+w_{j}(t),
$$

with the mixed-integer conditions:

$$
\begin{cases}0 \leq u_{g s}^{i j}(t) \leq M \alpha_{j}(t), & \forall i \text { with } M_{g s}(i, j) \neq 0, \\ 0 \leq u_{s d}^{j k}(t) \leq M\left(1-\alpha_{j}(t)\right), & \forall k \text { with } M_{s d}(j, k) \neq 0, \\ 0 \leq u_{s e}^{j}(t) \leq M\left(1-\alpha_{j}(t)\right), & \text { if } \exists j \text { with } M_{s e}(j) \neq 0,\end{cases}
$$

where $x_{j}(t) \in \mathbb{R}$ represents the amount of energy stored in $S_{j}$ at time step $t, u_{g s}^{i j}(t) \in$ $\mathbb{R}$ and $u_{s d}^{j k}(t) \in \mathbb{R}, u_{s e}^{j}(t) \in \mathbb{R}$ denote the charging and respectively the discharging components, $\sigma_{j}$ denotes the hourly self-discharge decay and the additive noise $w_{j}(t)$ accounts for the various sources of variation appearing in the storage dynamics (e.g., temperature influences, inverter performances and the like).

While presumably for certain storage units both charge and discharge operations are possible simultaneously, here we consider batteries which have a switched behavior (they function either in charge or discharge mode). This is modeled in the supplementary mixed-integer conditions $(8): \alpha_{j}(t) \in\{0,1\}$ is an auxiliary binary variable which switches between the modes of functioning. Specifically, when $\alpha_{j}(t)=1$ the battery is in discharge mode (since the input $u_{g s}^{i j}(t)$ is forced into zero) whereas, for $\alpha_{j}(t)=0$ the battery is in charge mode (since the outputs $u_{s d}^{j k}(t)$,

\footnotetext{
${ }^{2}$ Note that while $x_{j}(t)$ denotes the energy stored at time step $t$ the variables $u_{g s}^{i j}(t), u_{s d}^{j k}(t)$, $u_{s e}^{j}(t)$ and $w(t)$ are electrical powers which are multiplied by the sampling time $\Delta t=1$ hour. For a streamlined presentation, $\Delta t$ is hidden in (7) and in the following equations.
} 
$u_{s e}^{j}(t)$ are forced into zero). The scalar $M$ is an appropriately chosen constant (i.e., significantly larger than the rest of the variables and playing the role of a relaxation constant).

\section{Storage constraints}

Each storage unit has bounds on the quantity of stored energy

$$
B_{\min }^{j} \leq x_{j}(t) \leq B_{\max }^{j}
$$

where $B_{\text {min }}^{j}, B_{\text {max }}^{j} \in \mathbb{R}$ and on the rate of charge/discharge at time $\mathrm{t}$

$$
V_{\min }^{j} \leq \Delta x_{j}(t) \leq V_{\max }^{j}
$$

where $V_{\text {min }}^{j}, V_{\text {max }}^{j} \in \mathbb{R}$ and $\Delta x_{j}(t)=x_{j}(t)-x_{j}(t-1)$ represents the charge/discharge variation.

Batteries have additional particularities, which stem from their physical characteristics. In particular, the minimum capacity, $B_{m i n}^{j}$, is determined from the Depth of Discharge (DoD), which is used to describe how deeply the battery can be discharged Diaf et al. (2007):

$$
B_{\min }^{j}=(1-D o D) B_{\max }^{j}
$$

In addition, the battery capacity $B_{\max }$ decreases as a function of time, usage and conditions of work, as defined by the relation:

$$
B_{\max }^{j}=\beta_{j}(t) \bar{B}_{\max }^{j}
$$

where $\bar{B}_{\text {max }}^{j}$ represents the nominal capacity of the battery and parameter $\beta_{j}(t) \in$ $(0,1)$ models the capacity decay and its value is generally empiric.

Storage cost

Depending on the type of storage unit, we may need to take into account wear and tear issues. While in the short-medium time point of view it makes sense to exploit completely the storage unit (i.e., the energy should flow to and from the storage unit at all times, to ensure that energy costs are minimized) in the longer time view it is counter-productive to over-use a component if the price of replacing it is bigger than the actual gains from its use. To account for this, depending on the nature of the energy storage component, different terms can be considered as 
penalties in the cost function:

$$
C_{s}(t)=\gamma_{s, d} \sum_{j=1}^{N_{s}} \alpha_{j}(t)+\gamma_{s, c} \sum_{j=1}^{N_{s}}\left(1-\alpha_{j}(t)\right)+\gamma_{s, s} \sum_{j=1}^{N_{s}}\left(1-\alpha_{j}(t)\right),
$$

where the first term penalizes repeated discharges, the second term penalizes repeated charges and the third penalizes the switch between charge/discharge. Depending on the numeric values given to the weights $\gamma_{s, d}, \gamma_{s, c}, \gamma_{s, s} \in \mathbb{R}$ we may penalize one or a combination of the criteria, i.e., charge, discharge, switch. For example, for a hidro pumping storage unit it would make sense to penalize the charging mode (as it takes power to operate the pump uphill); on the other hand, for a battery we may wish to minimize repeated cycles of charge/discharge ${ }^{3}$.

\subsection{Power generators}

\section{Generator dynamics}

Consider the generator system $G_{i}$ in Figure 2, whose dynamics can be described by:

$$
g_{i}(t+1)=f\left(g_{i}(t), v_{i}(t)\right), \quad i=1, \ldots, N_{g}
$$

where $f(\cdot, \cdot): \mathbb{R} \rightarrow \mathbb{R}$ represents the possibly nonlinear dynamics of the generator, $g_{i}(t) \in \mathbb{R}$ is the electrical power given by the generator and $v_{i}(t) \in \mathbb{R}$ denotes the internal and external parameters which influence the generator output.

In our control scheme we consider renewable generators and as such we may ignore the control aspects, the generator is assimilated to an external source of power which provides as much as possible given the external environment, e.g., wind velocity will decide the actual power output for a wind turbine generator.

The addition of photovoltaic dynamic systems, electrical vehicles (EV) and the like would entail some modifications in the constraints formulation, the optimization problem and the related simulation validation, with no conceptual changes from the methodological viewpoint.

\footnotetext{
${ }^{3}$ The battery cycle life is defined as the number of complete charge - discharge cycles a battery can perform before its nominal capacity falls below $80 \%$ of its initial rated capacity Divya and Østergaard (2009). Key factors affecting cycle life are time $t$ and the number of charge-discharge cycles completed. It is also important to mention that the cycle life depends on the Depth of Discharge (DoD) (11) and that it is assumed that the battery is fully charged and discharged each cycle. If the battery is only partially discharged each cycle, then, the cycle life can be larger: it is, therefore, important that DoD be clearly defined when specifying the cycle life.
} 


\section{Generator constraints}

The generator output can be sent to other various microgrid components: to the storage unit for further use, directly to the users or even to the external grid for selling. Since the electrical power sent to these components cannot be larger than the actual output, the following constraint is imposed:

$$
0 \leq \sum_{M_{g s}(i, j) \neq 0} u_{g s}^{i j}(t)+\sum_{M_{g d}(i, k) \neq 0} u_{g d}^{i k}(t)+\sum_{M_{g e}(i) \neq 0} u_{g e}^{i}(t) \leq g_{i}(t)
$$

with $\sum_{M_{g s}(i, j) \neq 0} u_{g s}^{i j}(t) \geq 0, \sum_{M_{g d}(i, k) \neq 0} u_{g d}^{i k}(t) \geq 0$ and $\sum_{M_{g e}(i) \neq 0} u_{g e}^{i}(t) \geq 0$.

\section{Generator profile}

As stated earlier, the wind turbine generator output mainly depends on external factors (in particular, the wind velocity). In order to have a good estimation of the output, we make use of wind profiles, as the ones obtained from real numerical data of a reliability test system Grigg et al. (1999).

For a wind turbine generator, $f(\cdot, \cdot)$ in $(14)$ is nonlinear, that is, the output of the generator depends nonlinearly on the wind speed.

Note that the wind profile is sampled at one hour interval which from the point of view of wind variation is quite large. For this reason and due to measurement uncertainties we have considered along the nominal wind profile a band of disturbances as illustrated in Figure 3a. Passing these values through the dynamics (14) of the generator we obtain a band of possible power output values, as illustrated in Figure 3b.

[Figure 3 about here.]

\subsection{Consumers}

\section{Consumer constraints}

A user $D_{k}$ in Figure 2 can consume electricity from three sources, i.e., the electrical storage, the power generator and the external grid. It is important to mention that the user demand of electricity, denoted as $d_{k}(t)$, need not be inelastic: that is, we may partition the consumer demand $d_{k}(t)$ into essential, $d_{e s}^{k}(t) \in \mathbb{R}$ and nonessential demand, $d_{n e s}^{k}(t) \in \mathbb{R}$, respectively ${ }^{4}$. Therefore, for a reliable management of the energy system it is necessary to ensure that at time $t$ the electricity purchased

\footnotetext{
${ }^{4}$ Note that when and if a power shortcoming occurs, we want to be able to safely cut from the non-essential part while still covering the essential part.
} 
by the consumers from the three sources will satisfy at least the essential demands. This translates into the following constraint:

$$
d_{e s}^{k}(t)<\sum_{M_{g d}(i, k) \neq 0} u_{g d}^{i k}(t)+\sum_{M_{s d}(j, k) \neq 0} u_{s d}^{j k}(t)+\sum_{M_{e d}(k) \neq 0} u_{e d}^{k}(t) \leq d_{e s}^{k}(t)+d_{n e s}^{k}(t) .
$$

Consumer demand profile

The consumer demand profile usually exhibits periodicity (both daily, weekly and seasonal) and can therefore be predicted with a good degree of accuracy as presented in detail in Prodan and Zio (2014a).

Note that the real profiles may differ from the nominal values due to external factors, uncertainty and so forth. Illustrations are given in Figure 4 where both the nominal profiles and the bands in which the real reference profiles lie are depicted.

[Figure 4 about here.]

Consumer cost

In the cost function, we penalize for the difference between actual and required demand:

$$
C_{d}(t)=\sum_{k=1}^{N_{d}} d_{e s}^{k}(t)+d_{n e s}^{k}(t)-\left[\sum_{M_{s d}(j, k) \neq 0} u_{s d}^{j k}(t)+\sum_{M_{g d}(i, k) \neq 0} u_{g d}^{i k}(t)+\sum_{M_{e d}(k) \neq 0} u_{e d}^{k}(t)\right]
$$

\subsection{External grid}

Electricity price profile

If the microgrid is not functioning in islanded mode, the price of electricity in the external grid, denoted as $e(t) \in \mathbb{R}$, is relevant in taking sell and buy decisions (sell when expensive and buy when cheap). Depending on the differing country rules, the electricity price might be pre-scheduled by an external authority or be decided by market pressures. In any case, we may make use of price profiles in order to optimize the energy usage. Such a profile, taken from Grigg et al. (1999) is illustrated in Figure $4 b$.

\section{External grid cost}

The electricity price will be used to penalize buying and encourage selling with the cost described by the following relation:

$$
C_{e}(t)=e(t) \cdot\left(\sum_{M_{e d}(k) \neq 0} u_{e d}^{k}(t)-\sum_{M_{g e}(i) \neq 0} u_{g e}^{i}(t)-\sum_{M_{s e}(j) \neq 0} u_{s e}^{j}(t)\right)
$$


where we make the simplifying assumption that both sell and buy prices are equal, without loss of generality.

\section{Optimization-based control for electrical storage scheduling}

The underlying goal of this paper is to provide a realistic control strategy for the microgrid system, and the storage scheduling in particular. Taking into account the dynamics, constraints, costs and profiles discussed in the previous section, we can now write a constrained optimization problem for the global microgrid system.

To do so let us define first the global state $\mathbf{x}(t)=\left[\begin{array}{lll}x_{1}(t)^{T}, \quad \ldots, & x_{N_{s}}(t)^{T}\end{array}\right]^{T} \in$ $\mathbb{R}^{N_{s}}$, which concatenates the states of the storage units ${ }^{5}$, the additive disturbance $\mathbf{w}(t)=\left[w_{1}(t), \ldots w_{N_{s}}(t)\right]^{T} \in \mathbb{R}^{N_{s}}$ and the input

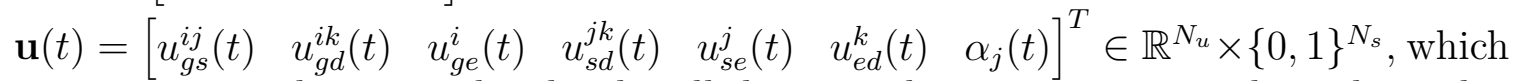
concatenates in lexicographical order all the control inputs appearing throughout the microgrid system ${ }^{6}$, with $N_{u}=N_{g s}+N_{g d}+N_{g e}+N_{s d}+N_{s e}+N_{e d}$ denoting the number of real valued control variables and $N_{g s}, N_{g d}, N_{g e}, N_{s d}, N_{s e}, N_{e d} \in \mathbb{R}$ as defined in (4)-(6).

Hence, with the above notation and the dynamic model of the storage unit detailed in (7), (8) we formulate the centralized system:

$\mathbf{x}(t+1)=\underbrace{\left[\begin{array}{ccc}1-\tau_{1} & & \\ & \ddots & \\ & & 1-\tau_{N_{s}}\end{array}\right]}_{\mathbf{A}} \mathbf{x}(t)+\underbrace{\left[\begin{array}{cc}\delta_{g s}^{b} & \mathbf{0}\end{array}\right]}_{\mathbf{B}_{c h}} \mathbf{u}(t)+\underbrace{\left[\begin{array}{llll}\mathbf{0} & \delta_{s d}^{b} & \delta_{s e}^{b} & \mathbf{0}\end{array}\right]}_{\mathbf{B}_{\text {disch }}} \mathbf{u}(t)+\mathbf{w}(t)$,

with matrices $\mathbf{A} \in \mathbb{R}^{N_{s} \times N_{s}} \mathbf{B}_{c h} \in \mathbb{R}^{N_{s} \times\left(N_{u}+N_{s}\right)}, \mathbf{B}_{\text {disch }} \in \mathbb{R}^{N_{s} \times\left(N_{u}+N_{s}\right)}$ decomposed in $\delta_{g s}^{b} \in \mathbb{R}^{N_{s} \times N_{g s}}, \delta_{s d}^{b} \in \mathbb{R}^{N_{s} \times N_{s d}}, \delta_{\text {se }}^{b} \in \mathbb{R}^{N_{s} \times N_{s e}}$ denoting hereafter the corresponding inputs from $\mathbf{u}(t)$ (such that (19) is equivalent with (7)) and the matrices $\mathbf{0}$ chosen with appropriate dimensions.

The dynamics (19) are subject to charge/discharge mixed-integer conditions (as in (8)), magnitude constraints (as in (9)), power variation (as in (10)), generator constraints (as in (15)), bounds on the energy transfer ${ }^{7}$, user demand validation (as

\footnotetext{
${ }^{5}$ Since these are the only microgrid units with internal dynamics.

${ }^{6}$ Note that the auxiliary binary variables $\alpha_{j}(t)$ appear as control inputs, since they model the charge and discharge operations of the storage units (see also Section 2.1).

${ }^{7}$ The constraints in (26) stand from physical limits on the energy transfer for each control signal,
} 
in (16)):

$$
\begin{aligned}
\mathbf{0} & \leq \mathbf{B}_{c h} \mathbf{u}(t) \leq M\left[\begin{array}{ll}
\mathbf{0} & I_{N_{s}}
\end{array}\right] \mathbf{u}(t), \\
\mathbf{0} & \leq \mathbf{B}_{\text {disch }} \mathbf{u}(t) \leq M\left[\begin{array}{ll}
\mathbf{0} & I_{N_{s}}
\end{array}\right](\mathbf{1}-\mathbf{u}(t)), \\
\mathbf{B}_{\min } & \leq \mathbf{x}(t) \leq \mathbf{B}_{\max }, \\
\mathbf{V}_{\min } & \leq \Delta \mathbf{x}(t) \leq \mathbf{V}_{\max }, \\
\mathbf{0} & \leq \mathbf{G u}(t) \leq \mathbf{g}(t), \\
\mathbf{d}_{e s}(t) & \leq \mathbf{D u}(t) \leq \mathbf{d}_{e s}(t)+\mathbf{d}_{\text {nes }}(t), \\
\mathbf{0} & \leq \mathbf{u}(t) \leq \overline{\mathbf{u}}(t) .
\end{aligned}
$$

More to the point, (20)-(21) describe in a compact form the battery charge/discharge conditions and (22)-(23) describe the battery magnitude and variation constraints with $\mathbf{B}_{\min }=\left[\begin{array}{lll}\ldots & B_{\min }^{j} & \ldots\end{array}\right]^{T}, \mathbf{B}_{\max }=\left[\begin{array}{llll}\ldots & B_{\max }^{j} & \ldots\end{array}\right]^{T}, \mathbf{V}_{\min }=\left[\begin{array}{lll}\ldots & V_{\min }^{j} & \ldots\end{array}\right]^{T}$, $\mathbf{V}_{\max }=\left[\begin{array}{lll}\ldots & V_{\max }^{j} & \cdots\end{array}\right]^{T}$, for all $j=1, \ldots, N_{s}$. Next, condition (24) describes the power distribution of the generator towards different sources with matrix $\mathbf{G}=$ $\left[\begin{array}{llll}\delta_{g s}^{g} & \delta_{g d}^{g} & \delta_{g e}^{g} & \mathbf{0}\end{array}\right] \in \mathbb{R}^{N_{s} \times\left(N_{u}+N_{s}\right)}$ decomposed in $\delta_{g s}^{g} \in \mathbb{R}^{N_{s} \times N_{g s}}, \delta_{g d}^{g} \in \mathbb{R}^{N_{s} \times N_{g d}}, \delta_{g e}^{g} \in$ $\mathbb{R}^{N_{s} \times N_{g e}}$ and $\mathbf{g}(t)=\left[\begin{array}{ll}\ldots & g_{i}(t)^{T}\end{array} \ldots\right]^{T}$, for all $i=1, \ldots, N_{g},(25)$ describes the necessary power distribution to the users with matrix $\mathbf{D}=\left[\begin{array}{lllllll}\mathbf{0} & \delta_{g d}^{d} & \mathbf{0} & \delta_{s d}^{d} & \mathbf{0} & \delta_{\text {ed }}^{d} & \mathbf{0}\end{array}\right] \in$ $\mathbb{R}^{N_{s} \times\left(N_{u}+N_{s}\right)}, \delta_{g d}^{d} \in \mathbb{R}^{N_{s} \times N_{g d}}, \delta_{s d}^{d} \in \mathbb{R}^{N_{s} \times N_{s d}}, \delta_{\text {ed }}^{d} \in \mathbb{R}^{N_{s} \times N_{e d}}, \mathbf{d}_{e s}(t)=\left[\begin{array}{ll}\ldots & d_{e s}^{k}(t)^{T}\end{array} \ldots\right]^{T}$, $\mathbf{d}_{\text {nes }}(t)=\left[\begin{array}{lll}\ldots & d_{e s}^{k}(t)^{T} & \ldots\end{array}\right]^{T}$, for all $k=1, \ldots, N_{d}$. Finally, condition (26) describes the physical limitations on the energy transfer between the different microgrid components with $\overline{\mathbf{u}}(t)=\left[\begin{array}{lllllll}\bar{u}_{g s}^{i j} & \bar{u}_{g d}^{i k} & \bar{u}_{g e}^{i} & \bar{u}_{s d}^{j k} & \bar{u}_{s e}^{j} & \bar{u}_{e d}^{k} & \mathbf{1}\end{array}\right]^{T}$.

The goal is to control the storage units such that the electricity taken from the external grid is minimized, the users demand is fulfilled, the electrical storages utilization is minimized and the generators utilization is maximized. For a reliable microgrid energy management we consider the recursive construction of an optimal open-loop control sequence $u=\left\{\mathbf{u}(t), \mathbf{u}(t+1), \cdots, \mathbf{u}\left(t+N_{\mathrm{p}}-1\right)\right\}$ over a finite constrained receding horizon $N_{p}$, which leads to a feedback control policy by the effective

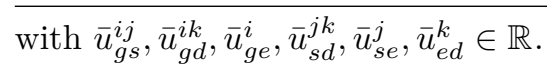


application of the first control action as system input:

$$
\mathbf{u}^{*}=\arg \min _{\mathbf{u}(t), \mathbf{u}(t+1), \cdots, \mathbf{u}\left(t+N_{\mathrm{p}}-1\right)} \sum_{l=0}^{N_{\mathrm{p}}-1} \gamma^{l} \cdot C(t+l),
$$

subject to the set of constraints defined in (20)-(26) for $t=0, \ldots, N_{p}-1$.

In (27) the length of the prediction horizon is denoted by $N_{p}, \gamma \in(0,1)$ represents a weighting parameter and the cost function is the sum of the previously formulated cost functions in (18), (17) and (13):

$$
C(t)=\gamma_{e} C_{e}(t)+\gamma_{d} C_{d}(t)+\gamma_{s} C_{s}(t)
$$

with $\gamma_{e}, \gamma_{d}, \gamma_{s} \in \mathbb{R}$ some constant parameters whose values reflect the importance given to the different terms of the cost. Using the above notations, the cost function can be written in a general form as:

$$
C(t)=(e(t) \mathbf{F}-\mathbf{D}) \mathbf{u}(t)+\mathbf{1}^{T}\left(\mathbf{d}_{e s}(t)+\mathbf{d}_{n e s}(t)\right)+\mathbf{H} \Delta \mathbf{u}(t),
$$

with $\mathbf{F}=\left[\begin{array}{llllll}\mathbf{0} & -\delta_{g e}^{f} & \mathbf{0} & \delta_{\text {se }}^{f} & \delta_{e d}^{f} & \mathbf{0}\end{array}\right]^{T} \in \mathbb{R}^{1 \times\left(N_{u}+N_{s}\right)}$ decomposed in $\delta_{g e}^{f} \in \mathbb{R}^{N_{g} \times N_{g e}}$, $\delta_{\text {se }}^{f} \in \mathbb{R}^{N_{s} \times N_{s e}}, \delta_{\text {ed }}^{f} \in \mathbb{R}^{1 \times N_{\text {ed }}}$ denoting the corresponding inputs from $\mathbf{u}(t)$ as previously mentioned, $\mathbf{H}=\left[\begin{array}{lllll}\mathbf{0} & \mathbf{0} & \ldots & \mathbf{0} & 1\end{array}\right]^{T}$ and $\Delta \mathbf{u}(t)=\mathbf{u}(t)-\mathbf{u}(t-1)$. The last term in (29) is chosen such that we penalize the charge/discharge cycle and assume there are no penalties for the actual charge and discharge operations (i.e., in (13) the weights become $\gamma_{s, d}=\gamma_{s, c}=0$ and $\gamma_{s, s}=1$ ).

Note that it remains a matter of choice on how to adjust the weights in order to decide the optimum usage of the storages. For example, a large $\gamma_{s}$ can lead to the battery being never used and hence its role being insignificant. On the other hand, too small a value leads to a premature capacity loss for the battery.

The profiles introduced in Section 2 appear as parameters here (e.g., the consumer load $d(t)$, the energy cost $e(t)$, etc.). The cost is variable in the sense that the weight matrix may change with time due to the variation in energy price ${ }^{8}$, but otherwise is linear in the input values. Therefore, we may denote this problem as one of MixedInteger Linear Programming (MILP). For this type of problems, efficient solvers exist and a reasonably large prediction horizon can be used Prodan et al. (2012).

Note also that the increase of the prediction horizon length in (27) means that the

\footnotetext{
${ }^{8}$ Note that we consider the same price for electricity selling and buying.
} 
optimization problem minimizes the cost along this entire horizon. The cost function, in turn, may be affected by uncertainties such that cost values further away along the prediction horizon are less reliable than the ones closer to the present. A solution is to assign in the optimization problem less importance to the cost values which are further in the future. This can be done by varying the weight $\gamma \in(0,1)$ in (27) associated to each cost value over the prediction horizon. The weight value decreases exponentially, with speed depending on the value taken by the rate $\gamma$ (see, for example Hovd and Braatz (2001)).

Up to this point, a nominal constrained optimization problem was considered. However, it is important to underline the fact that in real-life applications this is not sufficient as various issues can affect the system functioning (e.g., component malfunction and/or external perturbations). Therefore, in the following we discuss fault events and the subsequent redesign of the control scheme which allows to mitigate them. Several reasonable assumptions are made: the fault model is known; the fault is detected and isolated; the duration until repair and the duration between consecutive faults are known. The control scheme proposed hereafter has both robust and adaptive features:

- the fault duration is used to derive safe minimal capacity values for the storage units (such that they cover power failures during the fault event), i.e., a robust behavior;

- the constraints and costs appearing in the optimization problem are changed to guarantee acceptable performance (to the detriment of optimal performance), i.e., adaptive behavior.

\subsection{Fault tolerant control strategies}

Hereinafter, we consider external fault events affecting the renewable generators' power output. This means that they may fail to provide power to the battery, user and external grid (i.e., total output failure, $g_{i}(t) \rightarrow 0$ ). This can be modeled through the modification of the generator constraint (24) into:

$$
0 \leq \mathbf{G u}(t) \leq \mathbf{B}_{f} \mathbf{g}(t)
$$

where matrix $\mathbf{B}_{f}=\operatorname{diag}\left(\{0,1\}^{N_{g}}\right)$ characterizes the functioning of the generators. If all elements on the diagonal are ' 1 ' it means that all the generators are healthy and provide the expected power output. Whenever a fault occurs, one or more of the diagonal elements becomes ' 0 '. 
Since the fault detection and isolation reduces to inspecting the generators' outputs (and it is hence evident), what remains is to design the control reconfiguration strategies of the fault tolerant scheme. We aim at ensuring that the necessary quantity of energy is always stored in the electrical storage units such that, together with the external grid, the essential user demand for the k-th user is covered during the fault:

$$
\sum_{M_{s d}(j, k) \neq 0} B_{\min }^{j}(t) \geq \sum_{\tau=t}^{t+M T T R_{i}-1} \max \left[0, d_{e s}^{k}(\tau)-\bar{u}_{e d}^{k}-\sum_{M_{g d}(i, k) \neq 0, \mathbf{B}_{f}(i, i) \neq 0} u_{g d}^{i k}(\tau)\right],
$$

with $M T T R_{i}$ (Mean Time to Repair) representing the expected fault duration for the i-th generator ${ }^{9}$. Relation (31) constraints the storage units' energy level to a minimum safe level. That is, assuming a certain fault, the remaining power sources (external grid, remaining generators, storage units) have to cover the essential demand of the users. In this sense, we have that the storage units need to store enough energy to compensate for the fallen generator for the duration of the fault.

In fact, during this time we may consider that the remaining healthy generators send all their power to the users:

$$
\sum_{k=1}^{N_{d}} u_{g d}^{i k}(\tau)=g_{i}(\tau), \forall i=1, \ldots, N_{g}, \forall \tau=t, \ldots, t+M T T R_{i}-1,
$$

and $M_{g d}(i, k) \neq 0, \mathbf{B}_{f}(i, i) \neq 0$ in $(30)$.

Using (31) and (32) and considering the capacity bounds specific to the storage units we have an optimization problem whose output are the profiles of minimum storage which ensures essential demand coverage:

$$
\begin{aligned}
B_{h, \text { min }}^{1}(t) \ldots B_{h, \min }^{N_{s}}(t)= & \min _{B_{\min }^{1}(t) \ldots B_{\min }^{N_{s}}(t)} \sum_{j=1}^{N_{s}} B_{\min }^{j}(t) \\
& \text { such that }(31) \text { and }(32) \text { are verified and } \\
& (1-D O D) B_{\max }^{j} \leq B_{\min }^{j}(t) \leq B_{\max }^{j}, \forall j=1, \ldots, N_{s} .
\end{aligned}
$$

Furthermore, denoting $\mathbf{B}_{h, \min }(t)=\left[B_{h, \min }^{1}(t) \ldots B_{h, \min }^{N_{s}}(t)\right]$ the optimization problem

\footnotetext{
${ }^{9}$ If condition (31) can be interpreted as a passive (robust) approach, then satisfying only the user essential demands as in (16) can be considered as an active (adaptive) measure.
} 
(33) can be rewritten in a compact form:

$$
\begin{aligned}
\mathbf{B}_{h, \min }(t)= & \min _{\mathbf{B}_{h, \min }(t)} \mathbf{B}_{\min }(t) \mathbf{1}^{T} \\
& \text { such that }(31) \text { and }(32) \text { are verified and } \\
& (1-D O D) B_{\max }^{j} \leq B_{\min }^{j}(t) \leq B_{\max }^{j}, \forall j=1, \ldots, N_{s} .
\end{aligned}
$$

Remark 1. Note that in the above equations we made a series of simplifying assumptions. Firstly, we assume that there is a single generator under fault and that the fault is persistent. Secondly, we assume that the energy to be stored will never surpass the actual storage capacity (i.e., $B_{\min }^{j}(t) \leq B_{\max }^{j}$ ). Since ensuring that the storage units are large enough is a design choice, we assume that their capacity is large enough to accommodate (34). Conversely, we may use this information to see how long can the microgrid function in a safe mode (without unacceptable performance degradation, i.e., how long the storage units can guarantee essential demand satisfaction).

[Figure 5 about here.]

Condition $\mathbf{x}(t)>\mathbf{B}_{\min }(t)=\mathbf{B}_{h, \min }(t)$ is the capacity bound we aim to ensure at all times but it cannot be respected when under fault or immediately after. Hence, three modes of functioning are identified: i) nominal functioning after a previous fault, ii) steady state nominal functioning and iii) under fault. In the following, for thoroughly analyzing the microgrid modes of functioning under generator failures, we make use of the proof of concept illustration in Figure 5 where a typical functioning scenario is depicted: at $t_{0}$ the microgrid enters into faulty functioning due to the $i$-th generator having an outage. This continues up to time $t_{1}$ when the fault is solved and the microgrid regains its healthy functioning. The next time instants $t_{2}$ and $t_{3}$ retrace the same scenario (fault and subsequent recovery). Therefore, the three modes of functioning which provide the solution of safe stopping the microgrid system are:

- Nominal functioning after a previous fault: the battery is empty or close to empty due to its usage during the recovery phase (ended at $t_{1}$ as in Figure 5); the goal is, then, to charge it as fast as possible in order to reach the safe storage capacity obtained in (33). This cannot be done instantaneously due to practical limitations (charge variation bounds for example). Hence, we need first to determine a feasible recharging interval denoted as $N_{\text {fill }} \in \mathbb{R}$. This can 
be done e.g., by solving a minimal time problem:

$$
\begin{aligned}
N_{\text {fill }}=\min & \tau \\
& \text { such that } \mathbf{x}\left(t_{1}+\tau\right) \geq \mathbf{B}_{h, \min }\left(t_{1}+\tau\right), \text { and } \\
& \text { equations }(19)-(26) \text { are verified for } t=t_{1}, \ldots, t_{1}+\tau .
\end{aligned}
$$

We can now either provide condition $\mathbf{x}\left(t_{1}+N_{\text {fill }}\right) \geq \mathbf{B}_{h, \text { min }}\left(t_{1}+N_{\text {fill }}\right)$ as a terminal constraint (which is not obvious since $t_{1}+N_{\text {fill }}$ will probably be beyond the prediction horizon) or with a suboptimal approach, by forcing $\mathbf{B}_{\min }(t)$ to increase towards the expected safe value $\mathbf{B}_{h, \min }(t)$ in the filling time $N_{\text {fill }}$ along a linear mapping:

$$
\mathbf{B}_{r, \min }(\tau)=\mathbf{B}_{\text {min }}\left(t_{1}\right)+\frac{\tau-t_{1}}{N_{f i l l}}\left(\mathbf{B}_{h, \min }\left(t_{1}+N_{f i l l}\right)-\mathbf{B}_{\text {min }}\left(t_{1}\right)\right),
$$

for all $\tau=t_{1}, \ldots, t_{1}+N_{\text {fill }}$.

Thus, in the transitional period we will increase the minimum storage requirement step by step ${ }^{10}$ :

$$
\mathbf{x}(t)>\mathbf{B}_{\text {min }}(t)=\mathbf{B}_{r, \min }(t), \forall t=t_{1}, \ldots, t_{1}+N_{f i l l} .
$$

- Steady state nominal functioning: sufficient time has passed since the last fault. The battery already verifies (31) at the current instant and has to verify it for all future instances of the prediction horizon:

$$
\mathbf{x}(t)>\mathbf{B}_{\text {min }}(t)=\mathbf{B}_{h, \min }(t), \forall t>t_{1}+N_{\text {fill }}
$$

- Under fault: we enforce only the physical minimal storage requirements (11) for the interval $t=t_{0}, \ldots, t_{1}$.

$$
\mathbf{x}(t)=\mathbf{B}_{\text {min }}(t)=\mathbf{B}_{f, \min }(t) .
$$

The above remarks and constructions can be synthesized in algorithm Algorithm 1 which is to be followed by a supervisor unit.

Since we wish that in general the load demands are respected, we can transform

\footnotetext{
${ }^{10}$ If $N_{\text {fill }}<\min M T T F_{i}$ we have leeway to choose a larger $N_{f i l l}$. This will soften the storage requirements.
} 


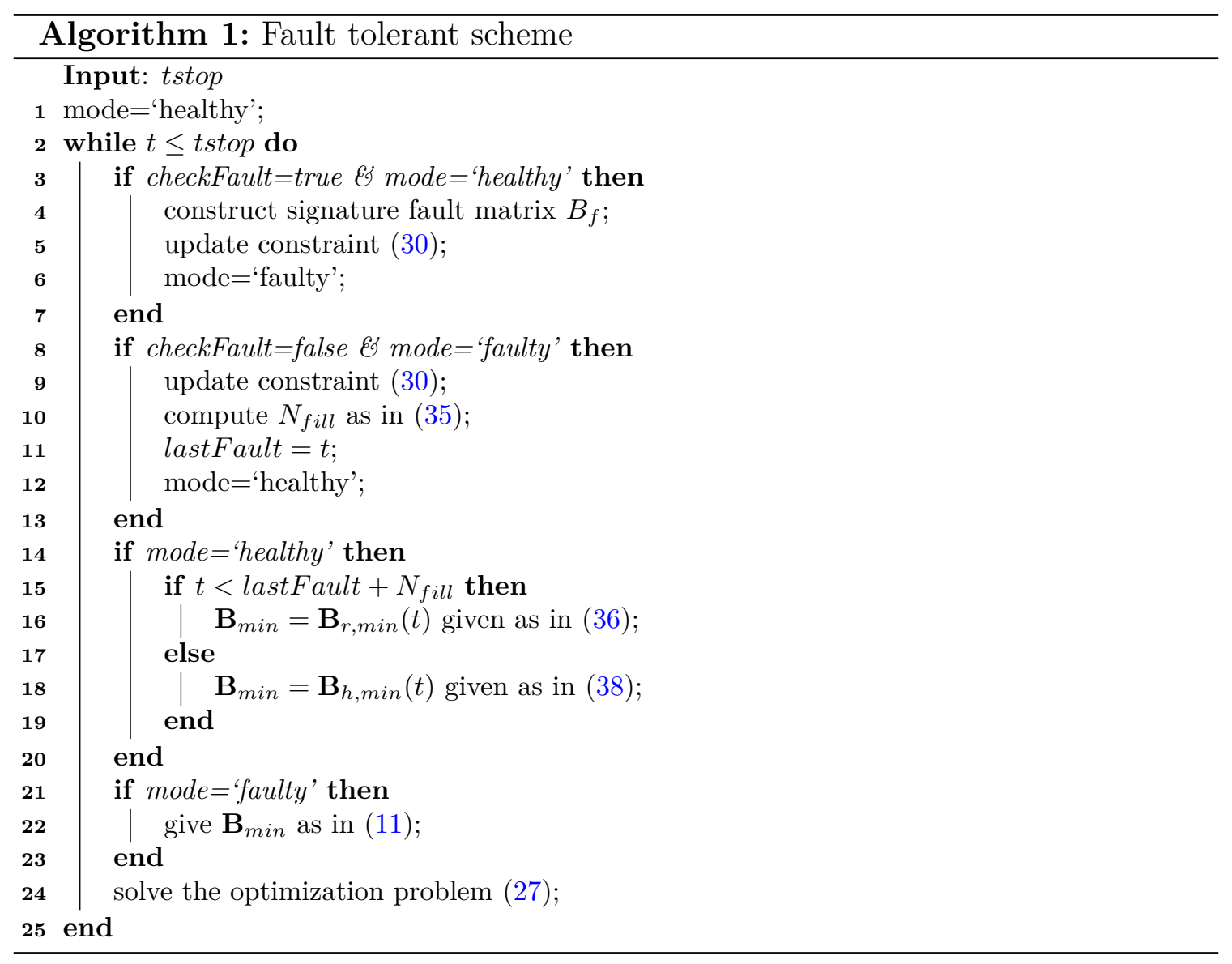


constraint (16) into a "soft constraint" through the addition of a slack variable. In the following subsection this issue is discussed in detail.

\subsection{Soft constraints}

The primordial requirement of the microgrid scheme is to fulfill the user demands as best as the microgrid components allow. To this end, we made use of constraint (18) to force at all times the coverage of the essential demand and of the cost (25) to tilt the control towards providing complete user coverage (both essential and nonessential). The issue in this approach is that choosing the weight $\gamma^{d}$ associated to the cost in (28) makes the difference between covering only the essential demand or both essential and non-essential demand (i.e., if too low relatively to the other weights, the optimization problem chooses to provide only essential demand, if it is too large, numerical issues may appear).

To correctly characterize the weight $\gamma^{d}$, we make use of the "hard/soft constraints" notion. Basically, we consider that the original "hard constrained" problem forces the validation at all times of equality

$$
\mathbf{D u}(t)=\mathbf{d}_{e s}(t)+\mathbf{d}_{n e s}(t)
$$

In this case, there may be initial conditions such that there exists no input sequence for which the problem is feasible ((40) cannot be validated). In such a situation the optimization solver will find no solution and consequently will not provide any input for the microgrid. This is in general considered unacceptable and practical MPC implementations therefore include some way of relaxing the constraints to ensure that the optimization problem is always feasible. We proceed here by adding slack variables $\epsilon^{k}(t) \geq 0$, which make the constraints (40) "soft":

$$
\begin{aligned}
\mathbf{d}_{e s}(t)+\mathbf{d}_{n e s}(t) & \leq \mathbf{D u}(t) \\
\operatorname{Du}(t)+\epsilon(t) & =\mathbf{d}_{e s}(t)+\mathbf{d}_{n e s}(t), \quad \epsilon(t) \geq 0
\end{aligned}
$$

When using soft constraints, the MPC formulation adds the variables $\epsilon(t)$ in the constraint equations which allow relaxing some of the constraints, while the optimization cost function includes terms which penalize the constraint violation:

$$
C_{d}(t)=\gamma^{d}\|\epsilon(t)\|_{1}
$$

The conditions of exact correspondence between "hard" and "soft" constraints follow classic optimization results Fletcher (1987) and express conditions on the Lagrangian multipliers of the original optimization problem (due to the fact that we have "soft- 
ened" only constraints (40) we consider only the associated Lagrangians):

$$
\gamma^{d} \geq \max _{\mu_{d}}\left\|D^{T} \mu_{d}\right\|_{\infty} .
$$

Finding the minimum for problem (44) can be done by solving a bi-level optimization problem, e.g., via a MILP formulation as in Hovd and Stoican (2013).

\section{Simulation results}

In this section, we introduce an example of a microgrid and the simulation results for its operation and control. The forthcoming simulations use the numerical data of a test system (IEEE RTS-96) developed for bulk power system reliability evaluation studies Grigg et al. (1999).

[Figure 6 about here.]

Consider the microgrid of Figure 6 which contains $N_{g}=2$ generators, $N_{s}=2$ electrical storage units and $N_{d}=2$ consumers. All components are characterized by the dynamic models, profiles, constraints and costs presented in detail in Section 2. The microgrid system is connected to the main utility grid through a transformer. Here, the transformer provides electrical power from the external grid as well as information about the electrical market price, which plays an important role as the storage units may decide to keep or use the stored energy depending on the external price. The consumers have also the possibility to take electrical power from the external grid when the renewable resources are not available (or sufficient).

The interactions between the independent components of the microgrid are most important for accomplishing the consumers objectives. As shown in Figure 6 there are various links between the components of the microgrid, which determine the energy flow. The adjacency matrices characterizing the links between the microgrid components (see also Section 2) are:

$$
\begin{array}{ll}
M_{g s}=\left[\begin{array}{ll}
1 & 1 \\
0 & 1
\end{array}\right] ; \quad M_{g d}=\left[\begin{array}{ll}
0 & 0 \\
1 & 1
\end{array}\right] ; \quad M_{g e}=\left[\begin{array}{l}
1 \\
0
\end{array}\right] ; \\
M_{s d}=\left[\begin{array}{ll}
1 & 0 \\
0 & 1
\end{array}\right] ; \quad M_{s e}=\left[\begin{array}{l}
0 \\
0
\end{array}\right] ; \quad M_{e d}=\left[\begin{array}{ll}
1 & 1
\end{array}\right] ;
\end{array}
$$

with the corresponding number of non-zero entries $N_{g s}=3, N_{g d}=2, N_{g e}=1$, $N_{s d}=2, N_{s e}=0, N_{e d}=2$ as defined in (4)-(6). 
The global state of the microgrid system $\mathbf{x}(t)=\left[x_{1}(t)^{T}, x_{2}(t)^{T}\right]^{T} \in \mathbb{R}^{2}$ concatenates the states of the two storage units and the input

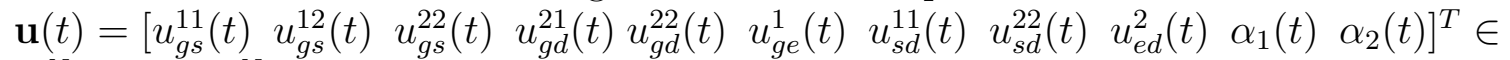
$\mathbb{R}^{N_{u}} \times\{0,1\}^{N_{s}}$, with $N_{u}=10, N_{s}=2$ concatenates all the control inputs of the microgrid system.

Using the general relation in (19) we obtain the following matrices which characterize the centralized system:

$$
\begin{aligned}
& \mathbf{A}=\left[\begin{array}{cc}
1-\sigma_{1} & 0 \\
0 & 1-\sigma_{2}
\end{array}\right] \in \mathbb{R}^{2 \times 2} ; \quad \mathbf{B}_{c h}=\left[\begin{array}{cc}
\delta_{g s}^{b} & \mathbf{0}
\end{array}\right] \in \mathbb{R}^{2 \times 12} \\
& \mathbf{B}_{\text {disch }}=\left[\begin{array}{lll}
\mathbf{0} & \delta_{s d}^{b} & \mathbf{0}
\end{array}\right] \in \mathbb{R}^{2 \times 12} \text { with } \quad \delta_{g s}^{b}=\left[\begin{array}{ccc}
1 & 0 & 0 \\
0 & 1 & 1
\end{array}\right], \quad \delta_{s d}^{b}=-I_{2} .
\end{aligned}
$$

The constraints (24), (25) are described by:

$$
\mathbf{G}=\left[\begin{array}{llll}
\delta_{g s}^{g} & \delta_{g d}^{g} & \delta_{g e}^{g} & \mathbf{0}
\end{array}\right] \in \mathbb{R}^{2 \times 12}, \quad \mathbf{D}=\left[\begin{array}{lllllll}
\mathbf{0} & \delta_{g d}^{d} & \mathbf{0} & \delta_{s d}^{d} & \mathbf{0} & \delta_{\text {ed }}^{d} & \mathbf{0}
\end{array}\right] \in \mathbb{R}^{2 \times 12},
$$

with $\delta_{g s}^{g}=\left[\begin{array}{lll}1 & 1 & 0 \\ 0 & 0 & 1\end{array}\right], \delta_{g d}^{g}=\left[\begin{array}{ll}0 & 0 \\ 1 & 1\end{array}\right], \delta_{g e}^{g}=\left[\begin{array}{l}1 \\ 0\end{array}\right], \delta_{g d}^{d}=\delta_{s d}^{d}=\delta_{e d}^{d}=I_{2}$.

The cost function (29) is described by:

$$
\begin{aligned}
& \mathbf{F}=\left[\begin{array}{llllll}
\mathbf{0} & -\delta_{g e}^{f} & \mathbf{0} & \delta_{\text {ed }}^{f} & \mathbf{0}
\end{array}\right]^{T} \in \mathbb{R}^{1 \times 12} \\
& \mathbf{H}=\left[\begin{array}{llllllllllll}
0 & 0 & 0 & 0 & 0 & 0 & 0 & 0 & 0 & 0 & 0 & 1
\end{array}\right]^{T} \in \mathbb{R}^{1 \times 12}
\end{aligned}
$$

with $\delta_{g e}^{f}=[-1], \delta_{e d}^{f}=\left[\begin{array}{ll}1 & 1\end{array}\right]$.

Considering the discretization step $\Delta t=1$ hour and the reference profiles described in Section 2 we provide in Table 3 the rest of the numerical values of the parameters used for the simulation results.

[Table 3 about here.]

Hereinafter, we consider a simulation horizon of 150 hours and depict in the following figures the profiles, states and control signals over the interval [50,150]. Any of the plotted signals is depicted through markers which show its value at each sampling time and the lines linking these markers characterize the value of the signal along the sampling interval. Depending on the control decisions taken, constructive details and assumptions made in the simulation, the plotted line can have a "stairwise" or "piecewise linear" shape. For example, the storage unit does 
not change the level of charge/discharge inside of a sampling interval and hence the value of the charge variation has a stairwise behavior. Accordingly, and since the storage unit has an affine dynamic, the storage level varies linearly along a sampling interval.

Figure 7 illustrates the charge level $x_{1}(t)$ and the charge variation $\Delta x_{1}(t)$ along the simulation horizon for the first storage unit (top and bottom figure respectively). To better underline the charge/discharge switching we also depict $\alpha_{1}(t)$, the binary signal which models the switching behavior. To have an uncluttered figure we scale and offset this signal (dotted blue line in the bottom figure). These three signals are linked as follows: if for the current sampling interval, the storage unit is in charge mode $\left(\alpha_{1}(t)=1\right)$ then there is a positive storage variation which reflects into an accompanying raise in the storage level. The converse holds for the discharge mode.

Not in the least, the bounds depicted in the top and bottom sub-figures (dashed black lines) represent the magnitude and variation constraints respectively, and it can be seen that they are respected.

Similarly, Figure 8 illustrates the charge stored in battery $x_{2}(t)$ along the same simulation horizon (i.e., 100 hours) as well as the charge variation $\Delta x_{2}(t)$, correspondingly to the binary variable codification $\alpha_{2}(t)$ and the constraints satisfaction.

[Figure 7 about here.]

[Figure 8 about here.]

Recall that the power flow through the microgrid is decided by the supervisor as a solution to the optimization problem (27). Therefore, the power flow can have unexpected characteristics and it is worthwhile to illustrate them.

In particular, it is interesting to illustrate the power produced by the generators and consumed by the storage units and the users; how a generator partitions its power output between users, storage units and external grid.

Figures 9-13 illustrate the various control signals of the microgrid. It can be seen that there is a complex interplay between the storage devices and the rest of the microgrid components. Particularly, at some instants, it appears that it is more efficient to give and take energy from the external grid rather than send it directly to the users.

Figure 9 illustrates the electrical power sent by the generators to the storage units. Note that the first generator sends to both storage units whereas the second sends only to the second storage unit.

[Figure 9 about here.] 
[Figure 10 about here.]

In Figure 10 we illustrate the same behavior but this time for the electrical power sent from the generators to the users and the external grid.

In Figures 9 and 10 we have shown different types of power flow between generators and the other microgrid components. In Figure 11 we now show which is the partitioning of the power flow of the first generator between all the other components in order to make clear that a generator may decide (depending on cost, constraints and its interconnections) to partition its power accordingly.

[Figure 11 about here.]

[Figure 12 about here.]

In Figure 12 we depict the power flows sent from the storage units to the users. As it can be seen, due to the optimization problem, the behavior appears to be "on-off": either the batteries are discharging to the maximum or they are in charge mode (there are relatively few charging values when the power sent is not close to maximal).

[Figure 13 about here.]

Figure 13 depicts the power received by both users from the external grid.

To better characterize the fulfillment of the demands of the users we depict in Figure 14 the demand profile (essential and total) of the first user against the actual received power. In the case of user 1 this means that the power flows from the second generator, first storage unit and the external grid. As it can be seen the essential demand is ensured at all times and, conditions permitting, the total demand is also covered.

\section{[Figure 14 about here.]}

Furthermore, to clarify the usage of the generators we illustrate in Figure 15 the power flows of interest for the first generator. The generator profile is depicted as a tube around the real power output $\left(g_{1}(t)\right.$ in solid black) and the actual power used in the migrogrid is shown as a dashed blue line (in the case of the first generator, the power output is partitioned between both storage units and the external grid). As it can be seen, due to constraints and cost imperatives at some time instants generator power is unused. 
[Figure 15 about here.]

In Figure 16 we show the minimal capacity of the storage units, $B_{\min }$ values computed as in Section 3.1 for a MTTR value of 5 hours and a total loss of power output in the second generator, $G_{2}\left(g_{2}(t) \rightarrow 0\right)$. As it can be seen in the figure, the second storage unit requires a large $B_{\min }$ value to cope with this fault scenario (it is getting in fact close to the $B_{\max }=6000 \mathrm{~W}$ value) whereas the first electrical storage requires no extra charge $\left(B_{\min }^{1}=0\right)$. These values are justified by the structure of the microgrid and by the fault's nature: a fault in the second generator cuts all the sources of charge for the first storage unit whereas the second unit can still draw power from the second generator which continues to function correctly.

[Figure 16 about here.]

Furthermore, to illustrate the microgrid behavior under fault we consider a total output fault affecting the second generator, $G_{2}$ during the time interval $[60,65]$ hours and a fault duration $M T T F=5$ hours. That is, during this interval no power is sent by the second generator to any of the grid components.

As discussed earlier, the fault event influences the control response and the minimal capacity bounds of the storage units. We differentiate between three cases: healthy (before the fault, the time interval $[50,59]$; after the fault and the recovery period, the interval $[86,150]$ ); faulty (time interval $[60,65]$ ) and recovery (time interval $[66,85]$ hours).

In Figure 17 we depict the relevant signals and explain their behavior (for clarity we illustrate them only during the time interval $[55,95]$ hours containing the fault event). The top-most figure illustrates the second generator output, and as expected, we observe that it drops to zero during the fault interval.

The second figure illustrates the behavior of the second storage unit, $S_{2}$ (we do not illustrate the same signals for the first storage unit due to space constraints and because the second is more relevant to the discussion). In this figure we illustrate $B_{h, m i n}^{2}(t), B_{m i n}^{2}(t)$ and $x_{2}(t)$. Recall that the stored energy $x_{2}(t)$ should stay above the robust safe minimal value $B_{h, \min }(t)$. This is indeed the case in the healthy time intervals $[50,59]$ and $[86,150]$. This is no longer the case while under fault or under recovery. This fact is illustrated by $B_{\min }$ which is the "actual minimal capacity" and which follows $B_{h, \min }$ when possible but goes to zero during the fault interval and then gradually increases during the recovery interval (we have chosen $N_{\text {fill }}=M T T R=20$ hours).

The two bottom-most figures illustrate the power received by the two users and their profile bounds (essential versus total power demanded). We point two elements: 
i) even under fault the essential demand is fulfilled at all times; ii) during the fault the users receive only the essential demand (such that the demands on the external grid, remaining generator and storage units are minimized).

[Figure 17 about here.]

\section{Conclusions}

This paper presented a reliable model predictive control approach for efficient energy storage and distribution in a microgrid system. Electricity costs, power consumption, generation profiles and power and energy constraints were taken into consideration in a Mixed-Integer Linear Programming optimization problem. Uncertainty due to variations in the environment (wind speed, load and electricity market price), failures and subsequent repairs of the generators as well as battery lifespan limitations are efficiently treated. Simulation results validate the proposed approach.

The original contributions are:

- by minimizing the charge/discharge cycles the battery life is increased: this is achieved through the analysis of the expected battery life/versus electricity prices to find the optimum balance between battery usage and profit gained from electricity management;

- by using mixed-integer techniques, we can model different situations and behaviors of the microgrid components (switching between functioning modes of the storage elements and the microgrid in general, connected and islanded);

- fault tolerant strategies are proposed in order to ensure the necessary amount of energy in the storage devices such that (together with the utility grid) the essential consumer demand is always covered;

- by applying these improvements, we obtain a Mixed-Integer Linear Programming (MILP) optimization problem, which is difficult to solve but still with acceptable computational efforts.

For future work, we envision to analyze interconnected microgrid systems and discuss the combination between multi-agent approaches, mixed-integer programming and centralized/distributed/decentralized algorithms for scheduling control. 


\section{Acknowledgments}

Ionela Prodan would like to acknowledge the support of AFFDU (Association Française des Femmes Diplomées des Universités) by awarding her with a "Bourse Dorothy LEET/AFFDU" 2014. Florin Stoican would like to acknowledge the Sectorial Operational Programme Human Resources Development 20072013 of the Ministry of European Funds through the Financial Agreement [grant number POSDRU/159/1.5/S/132395].

\section{References}

Bracco, S.and Delfino, F. P. F., Robba, M., Rossi, M., 2014. A mathematical model for the optimal operation of the university of genoa smart polygeneration microgrid: Evaluation of technical, economic and environmental performance indicators. Energy 64, 912-922.

del Real, A., Arce, A., Bordons, C., 2007. Hybrid model predictive control of a two-generator power plant integrating photovoltaic panels and a fuel cell. In: Proceedings of the 46th IEEE Conference on Decision and Control. pp. 5447-5452.

Diaf, S., Diaf, D., Belhamel, M., Haddadi, M., Louche, A., 2007. A methodology for optimal sizing of autonomous hybrid pv/wind system. Energy Policy 35 (11), $5708-5718$.

Divya, K., Østergaard, J., 2009. Battery energy storage technology for power systems - an overview. Electric Power Systems Research 79 (4), 511-520.

Fletcher, R., 1987. Practical Methods of Optimization. John Wiley $\backslash$ \& Sons, Inc.

Foley, A., Ó Gallachóir, B., Hur, J., Baldick, R., McKeogh, E., 2010. A strategic review of electricity systems models. Energy 35 (12), 4522-4530.

Grigg, C., Wong, P., Albrecht, P., Allan, R., Bhavaraju, M., Billinton, R., Chen, Q., Fong, C., Haddad, S., Kuruganty, S., 1999. The ieee reliability test system-1996. a report prepared by the reliability test system task force of the application of probability methods subcommittee. IEEE Transactions on Power Systems 14 (3), 1010-1020.

Hawkes, A., Brett, D., Brandon, N., 2009. Fuel cell micro-chp techno-economics: Part 1-model concept and formulation. International Journal of Hydrogen Energy 34 (23), 9545-9557. 
Hooshmand, A., Poursaeidi, M., Mohammadpour, J., Malki, H., Grigoriads, K., 2012. Stochastic model predictive control method for microgrid management. In: IEEE PESInnovative Smart Grid Technologies (ISGT). pp. 1-7.

Hovd, M., Braatz, R., 2001. Handling state and output constraints in mpc using time-dependent weights. In: American Control Conference, 2001. Proceedings of the 2001. Vol. 3. IEEE, pp. 2418-2423.

Hovd, M., Stoican, F., 2013. On the design of exact penalty functions for mpc using mixed integer programming. International Journal of Computers \& Chemical Engineering.

Johansson, B., 2013. Security aspects of future renewable energy systems-a short overview. Energy 61, 598-605.

Khalid, M., Savkin, A., 2010. A model predictive control approach to the problem of wind power smoothing with controlled battery storage. Renewable Energy 35 (7), $1520-1526$.

Liang, H., Zhuang, W., 2014. Stochastic modeling and optimization in a microgrid: A survey. Energies 7 (4), 2027-2050.

Lo Prete, C., Hobbs, B., Norman, C., Cano-Andrade, S., Fuentes, A., von Spakovsky, M., Mili, L., 2012. Sustainability and reliability assessment of microgrids in a regional electricity market. Energy 41 (1), 192-202.

Lund, H., 2007. Renewable energy strategies for sustainable development. Energy 32 (6), 912-919.

Negenborn, R., Houwing, M., De Schutter, B., Hellendoorn, J., 2009. Model predictive control for residential energy resources using a mixed-logical dynamic model. In: International Conference on Networking, Sensing and Control. pp. 702-707.

Parisio, A., Glielmo, L., 2011. Energy efficient microgrid management using model predictive control. In: Proceedings of the 50th IEEE Conference on Decision and Control and European Control Conference (CDC-ECC). pp. 5449-5454.

Pérez, E., Beltran, H., Aparicio, N., Rodriguez, P., 2013. Predictive power control for pv plants with energy storage. IEEE Transactions on Sustainable Energy 4 (2), $482-490$. 
Prodan, I., Stoican, F., Olaru, S., S.I., N., 2012. Enhancements on the Hyperplanes Arrangements in Mixed-Integer Techniques. Journal of Optimization Theory and Applications 154 (2), 549-572.

Prodan, I., Zio, E., 2014a. A model predictive control for reliable microgrid energy management. International Journal of Electrical Power and Energy Systems 61 (7), 399-409.

Prodan, I., Zio, E., 2014b. On the microgrid energy management under a predictive control framework. In: In Proceedings of the IEEE Multi-Conference on Systems and Control. IEEE, pp. $861-866$.

Qi, W., Liu, J., Christofides, P., 2012. Supervisory predictive control for long-term scheduling of an integrated wind/solar energy generation and water desalination system. IEEE Transactions on Control Systems Technology 20 (2), 504-512.

Rahimiyan, M., Baringo, L., Conejo, A., 2014. Energy management of a cluster of interconnected price-responsive demands. IEEE Transactions on Power Systems $29(2), 645-655$.

Rawlings, J., Mayne, D., 2009. Model Predictive Control: Theory and Design.

Sanseverino, E., Di Silvestre, M., Ippolito, M., De Paola, A., Lo Re, G., 2011. An execution, monitoring and replanning approach for optimal energy management in microgrids. Energy 36 (5), 3429-3436.

Tsikalakis, A., Hatziargyriou, N., 2011. Centralized control for optimizing microgrids operation. In: IEEE Power and Energy Society General Meeting. pp. 1-8.

Zervas, P., Sarimveis, H., Palyvos, J., Markatos, N., 2008. Model-based optimal control of a hybrid power generation system consisting of photovoltaic arrays and fuel cells. Journal of Power Sources 181 (2), 327-338. 


\section{List of Figures}

1 Microgrid energy management. . . . . . . . . . . . . . . 30

2 Interconnections in a typical microgrid system. . . . . . . . . . . 31

3 Reference profiles. (a) Wind speed profile and uncertainty band (b) Generator power profile and uncertainty band. . . . . . . . . . . 32

4 Reference profiles. (a) Consumer load profile and uncertainty band. (b) Electricity market price profile and uncertainty band. . . . . . . . 32

5 Fault tolerant strategy illustration. . . . . . . . . . . . . . 32

6 Microgrid architecture . . . . . . . . . . . . . . . 33

7 Battery 1 charge level and variation. . . . . . . . . . . 34

8 Battery 2 charge level and variation. . . . . . . . . . . . 35

9 Electrical power transmitted by the generators to electrical storage components. . . . . . . . . . . . . . . . 35

10 Electrical power transmitted by the generators to users. . . . . . . . . 36

11 Electrical power transmitted by the generator 1 to external grid and the two storage units. . . . . . . . . . . . . . . . 36

12 Electrical power transmitted by the storage units to users. . . . . . . 37

13 Electrical power transmitted by the external grid to users. . . . . . . 37

14 Electrical power transmitted by three sources to user 1. . . . . . . . 38

15 Electrical power consumed by user 1. . . . . . . . . . . . 38

16 Minimal capacity of the battery storage units. . . . . . . . . . . . 39

17 Microgrid system behavior under a fault event affecting generator $G_{2} . \quad 39$ 
Microgrid energy management

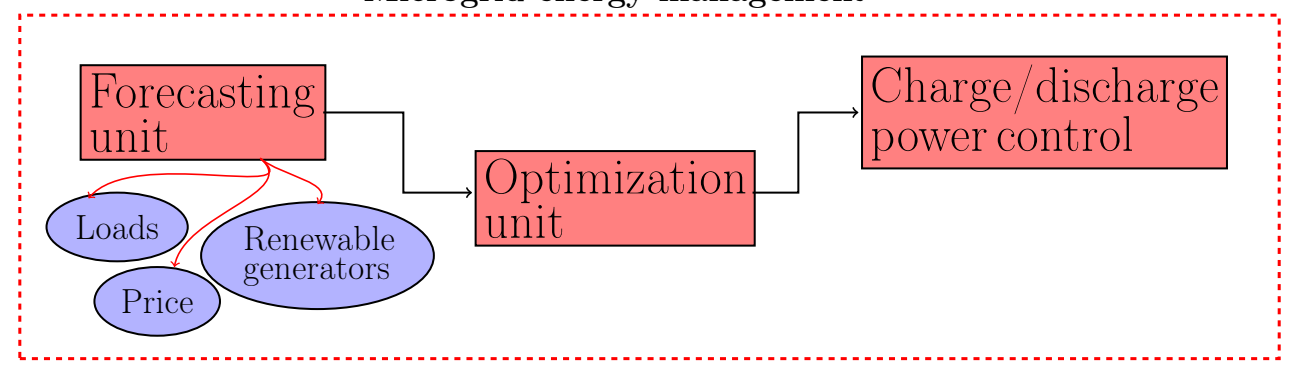

Figure 1: Microgrid energy management. 


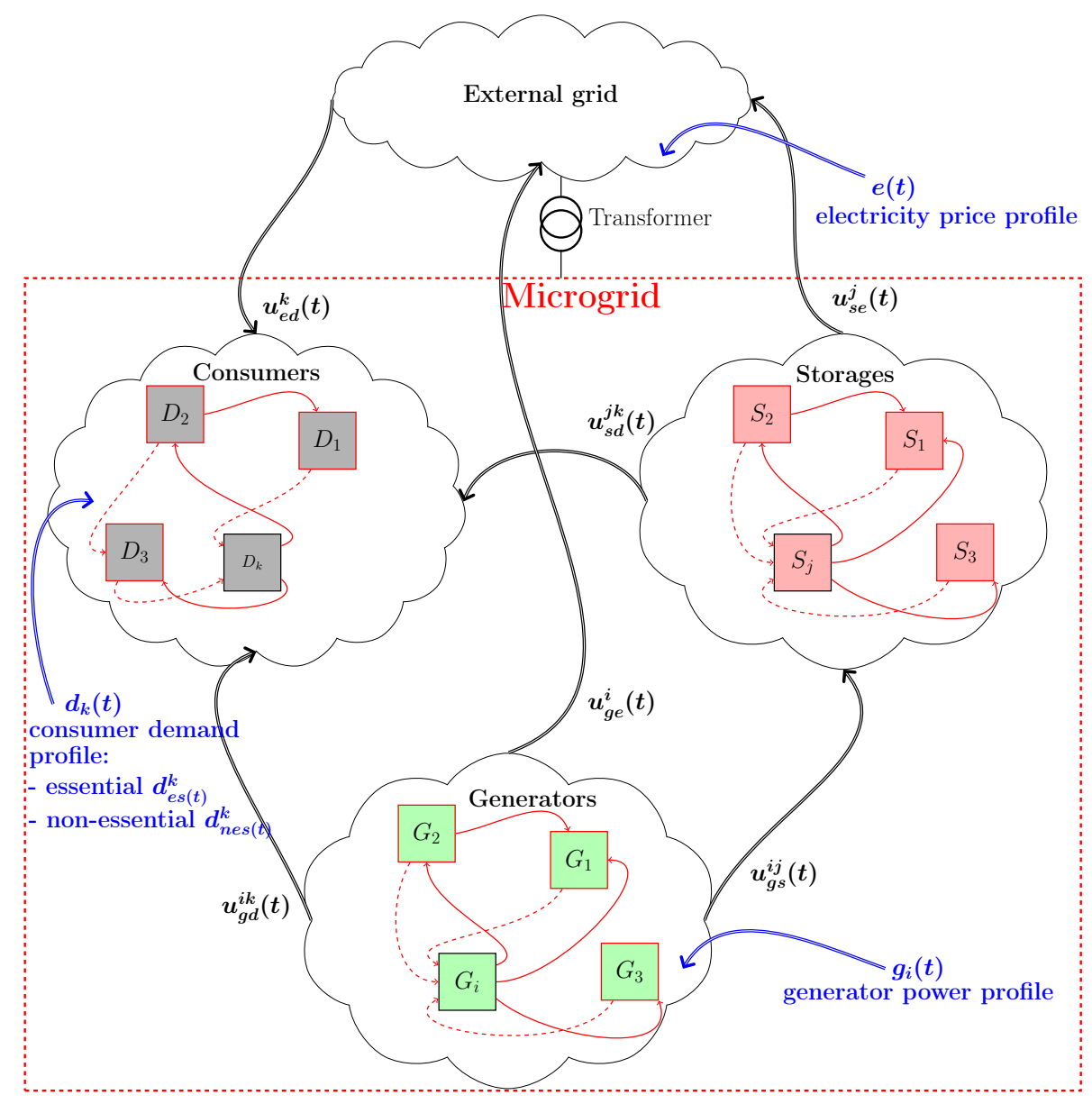

Figure 2: Interconnections in a typical microgrid system. 


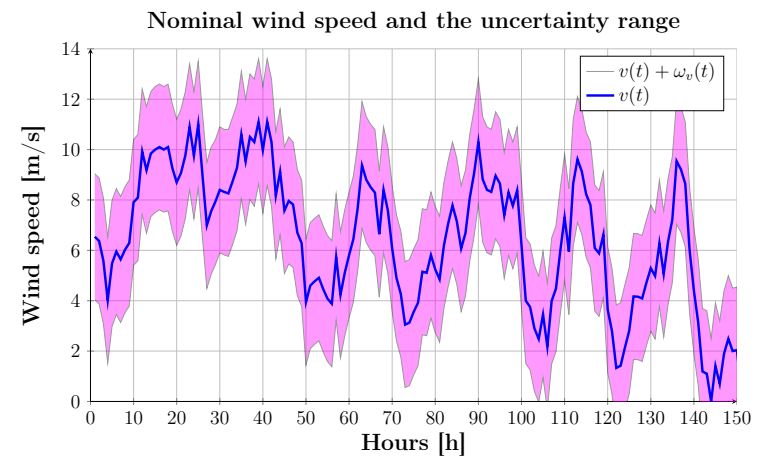

(a)

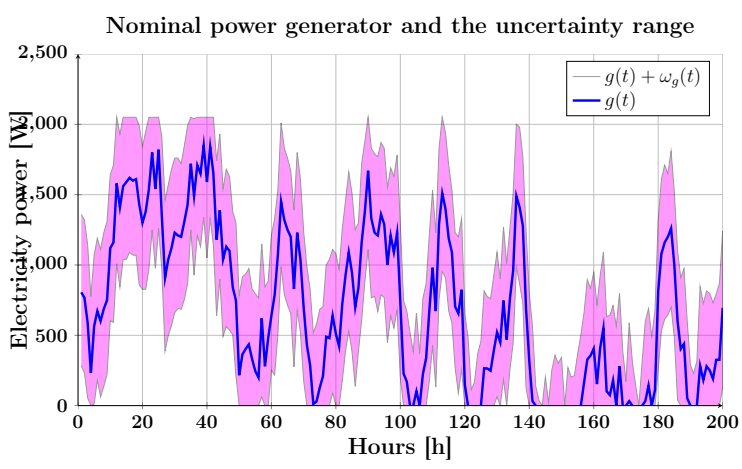

(b)

Figure 3: Reference profiles. (a) Wind speed profile and uncertainty band (b) Generator power profile and uncertainty band.

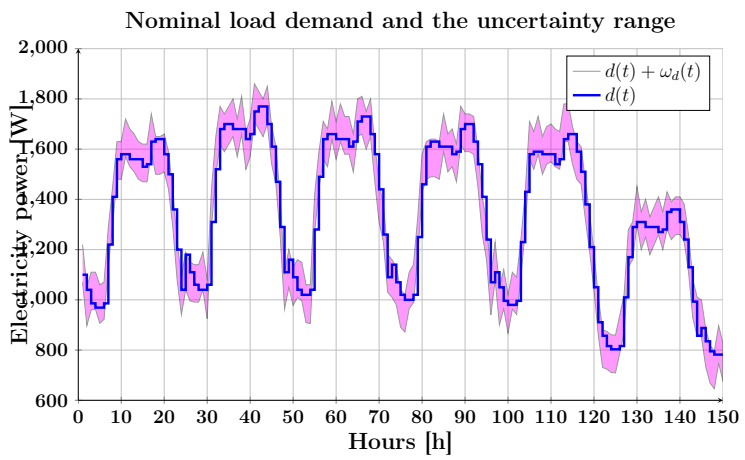

(a)

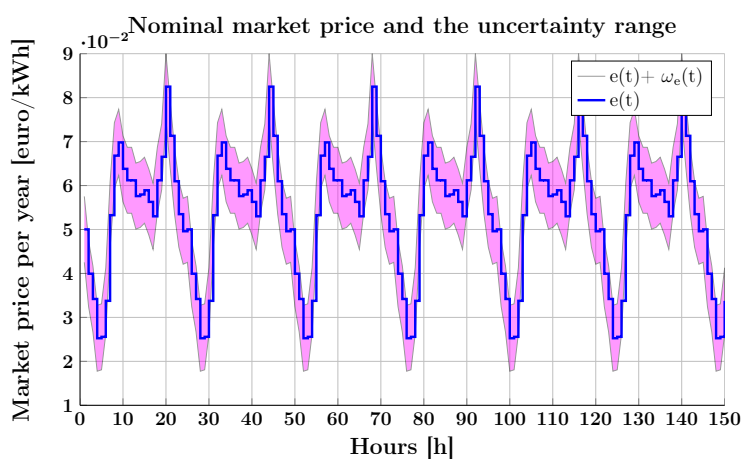

(b)

Figure 4: Reference profiles. (a) Consumer load profile and uncertainty band. (b) Electricity market price profile and uncertainty band.

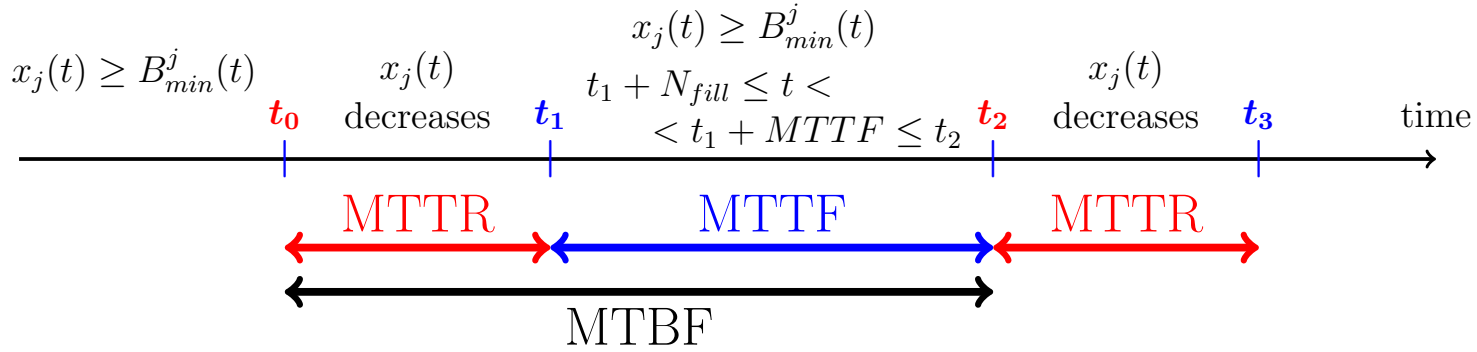

Figure 5: Fault tolerant strategy illustration. 


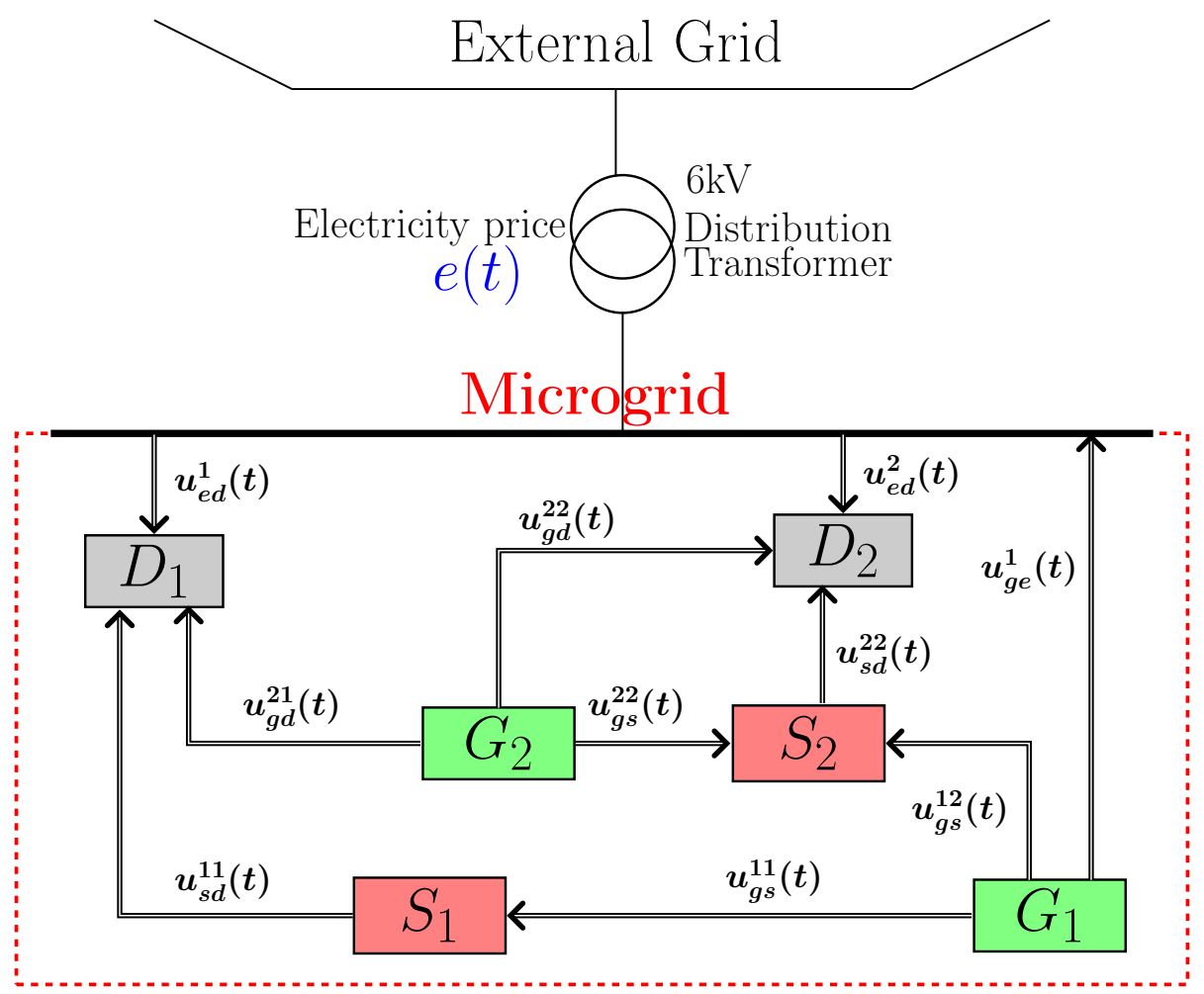

Figure 6: Microgrid architecture 

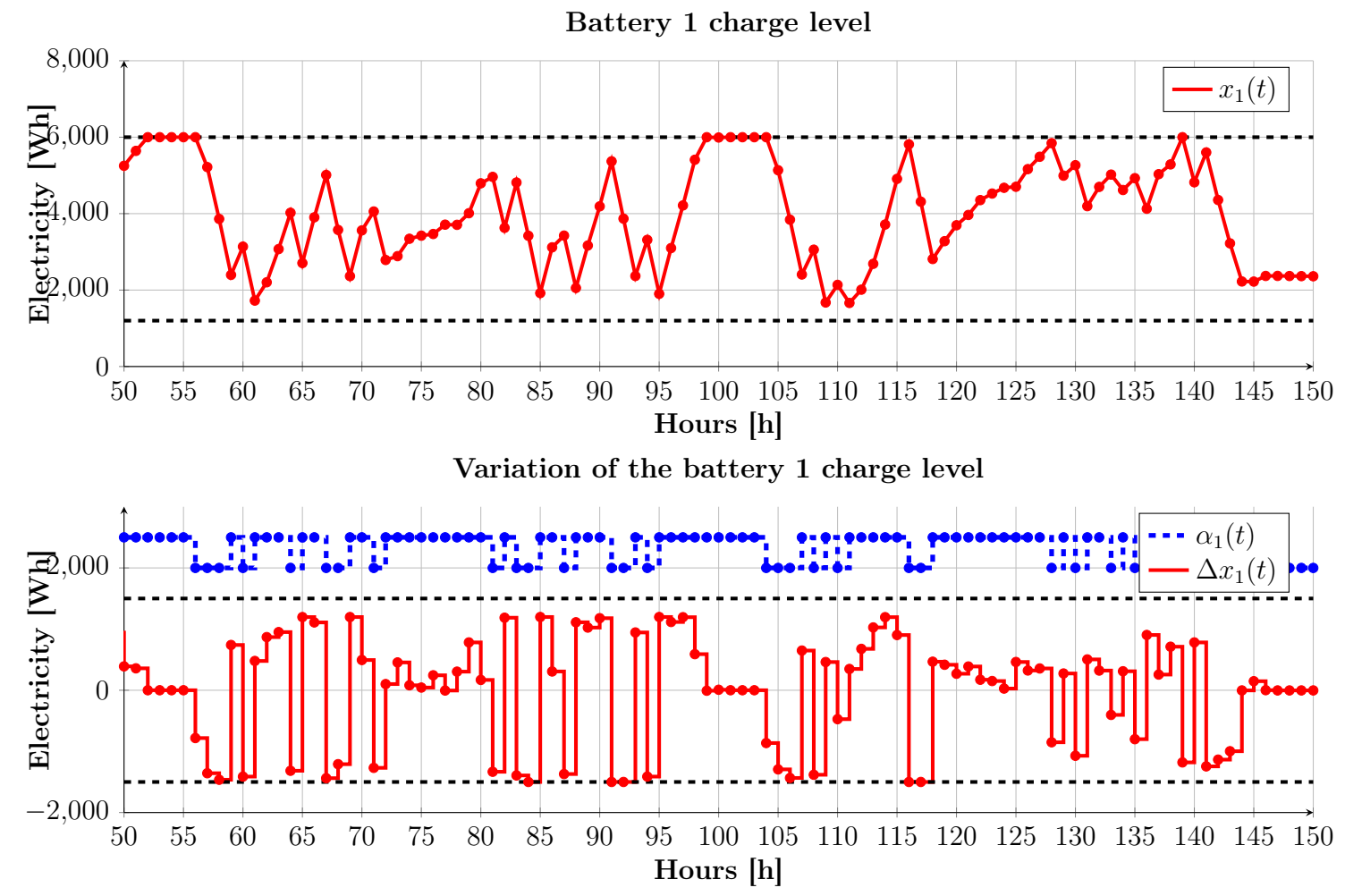

Figure 7: Battery 1 charge level and variation. 
Battery 2 charge level

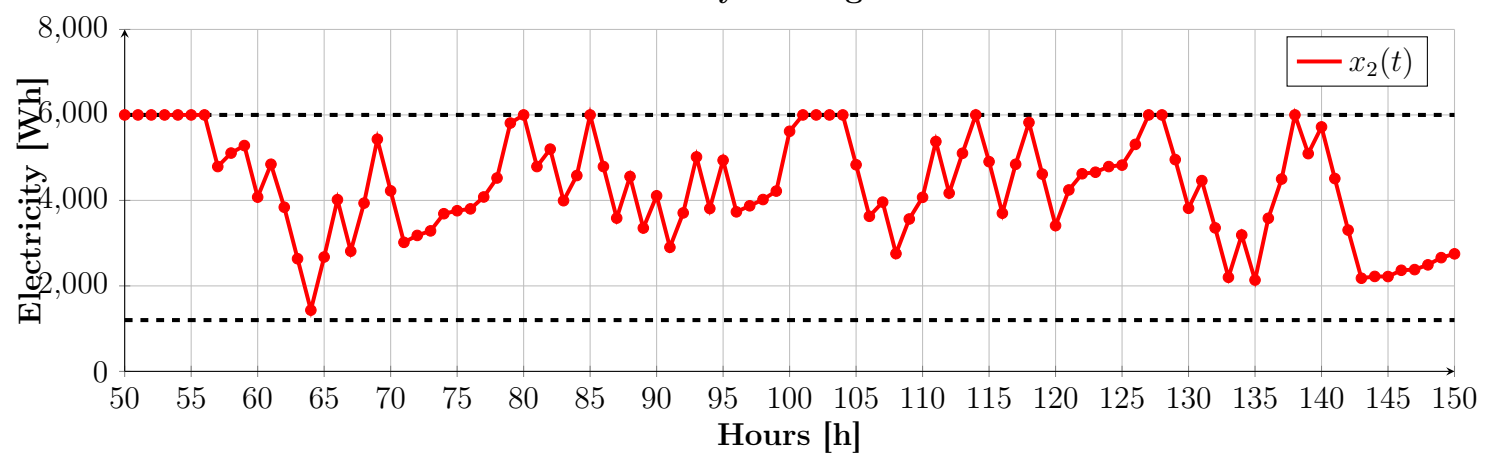

Variation of the battery 1 charge level

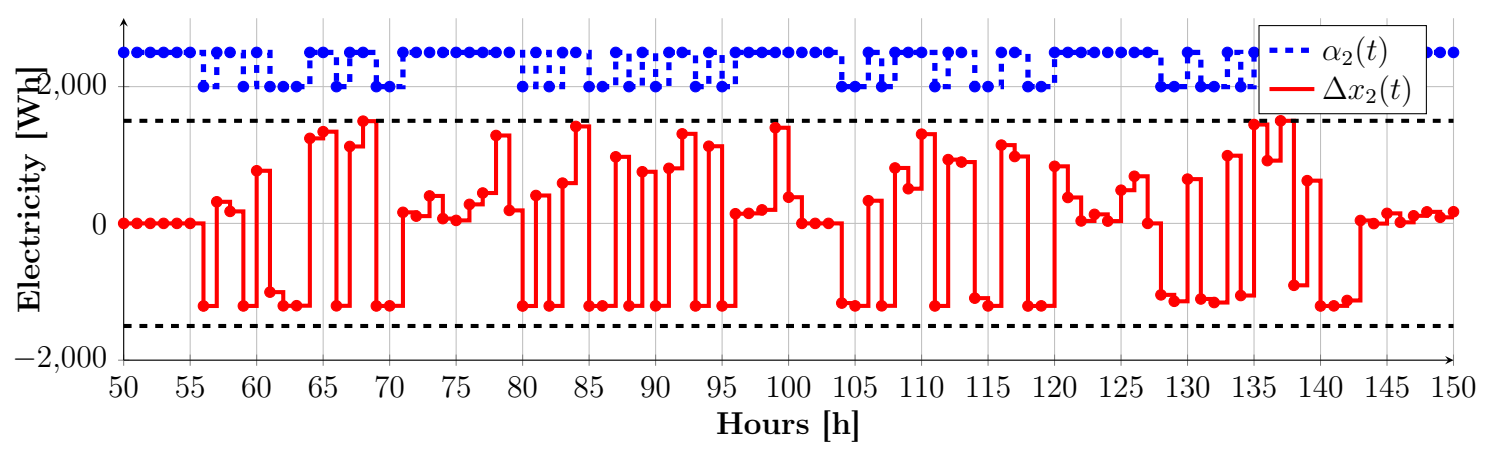

Figure 8: Battery 2 charge level and variation.

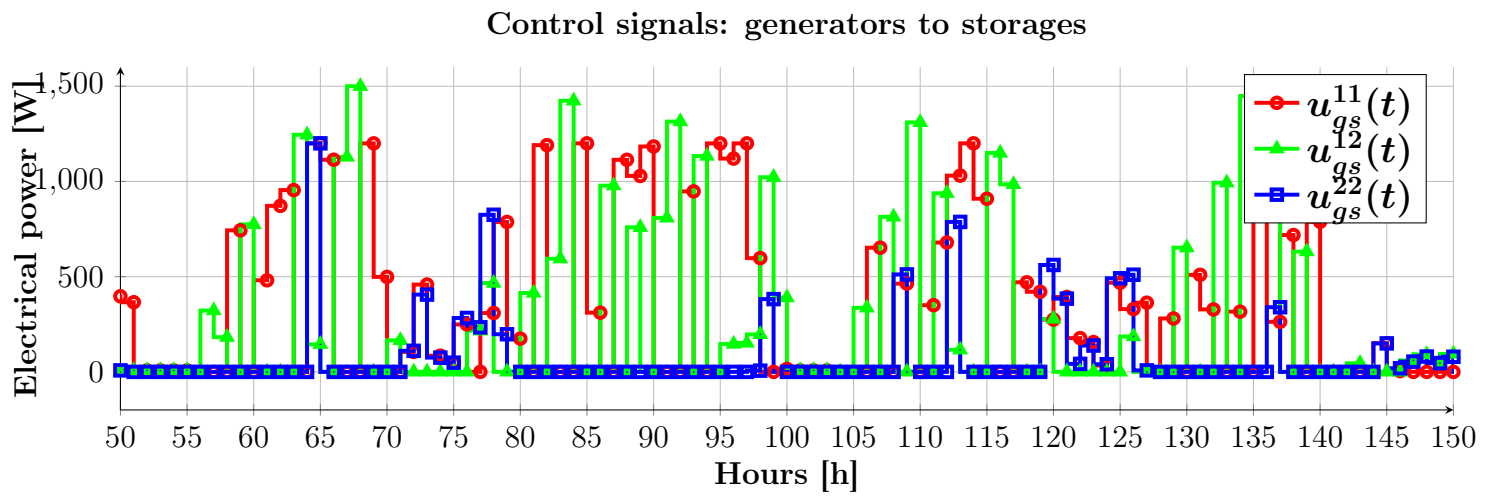

Figure 9: Electrical power transmitted by the generators to electrical storage components. 
Control signals: generators to users and external grid

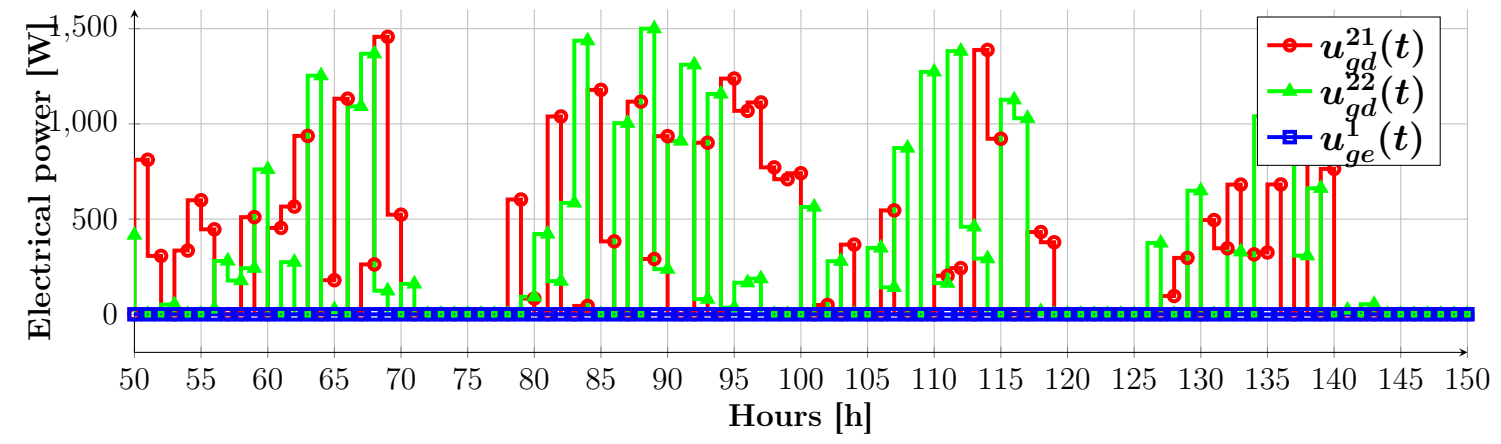

Figure 10: Electrical power transmitted by the generators to users.

Control signals: generator 1 to storage 1 , storage 2 and electrical grid

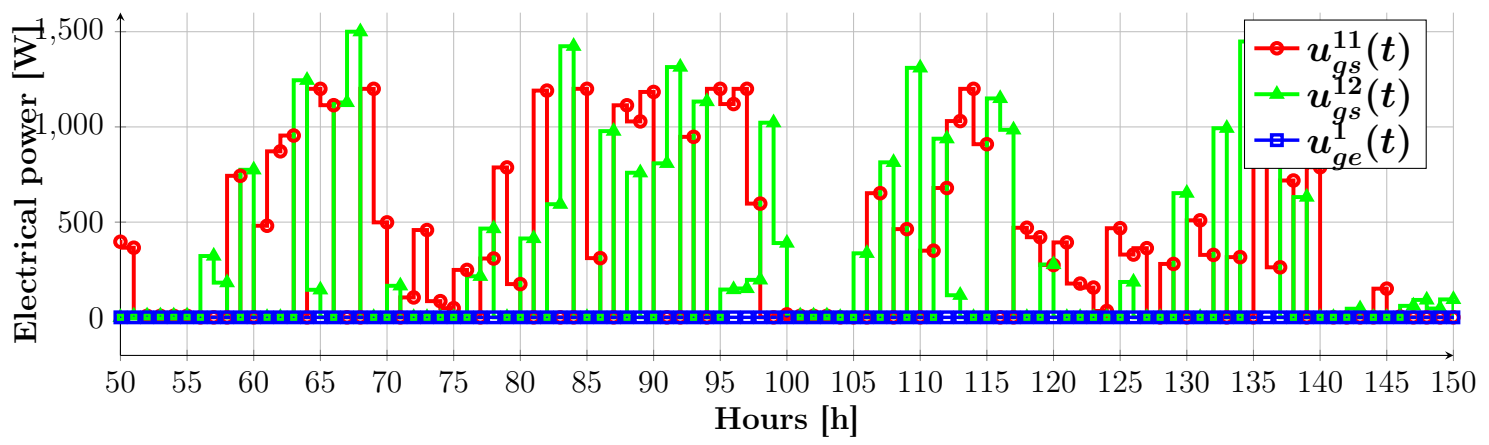

Figure 11: Electrical power transmitted by the generator 1 to external grid and the two storage units. 
Control signals: storages to users

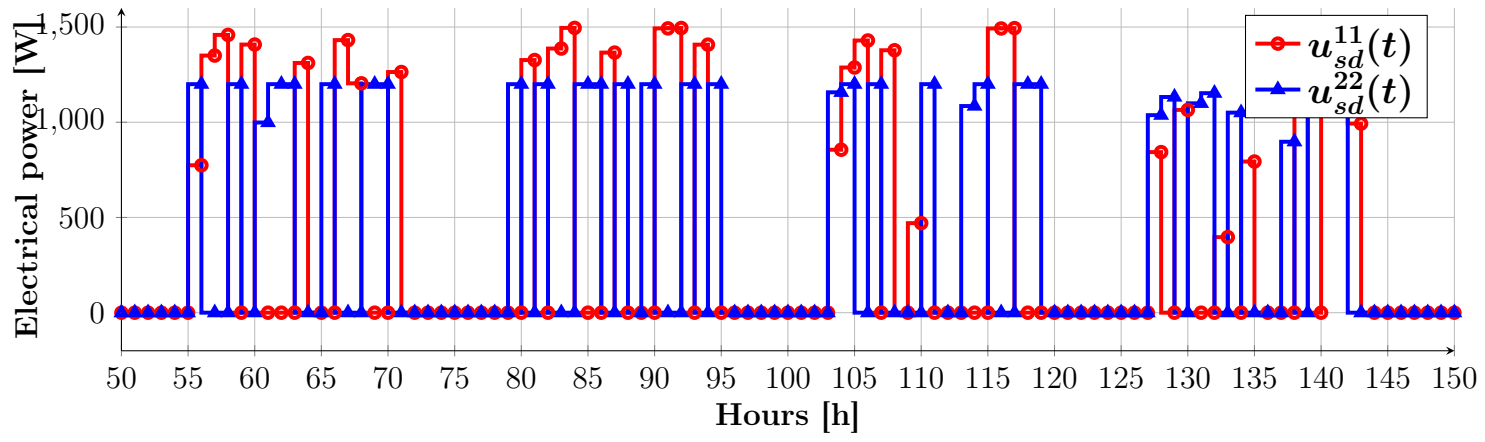

Figure 12: Electrical power transmitted by the storage units to users.

Control signals: external grid to users

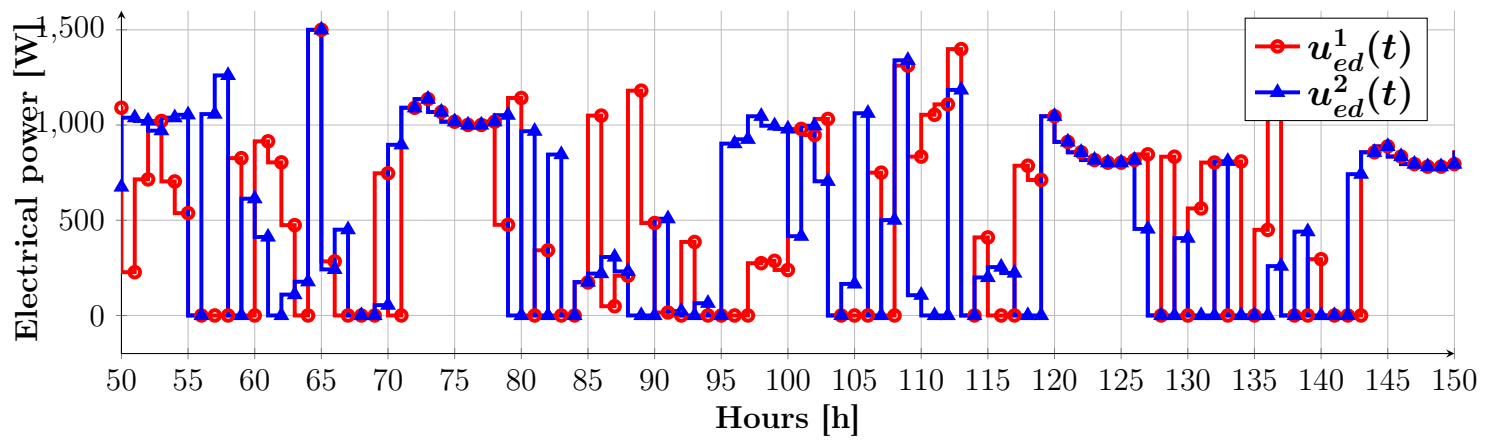

Figure 13: Electrical power transmitted by the external grid to users. 
User 1 demand profile and sources.

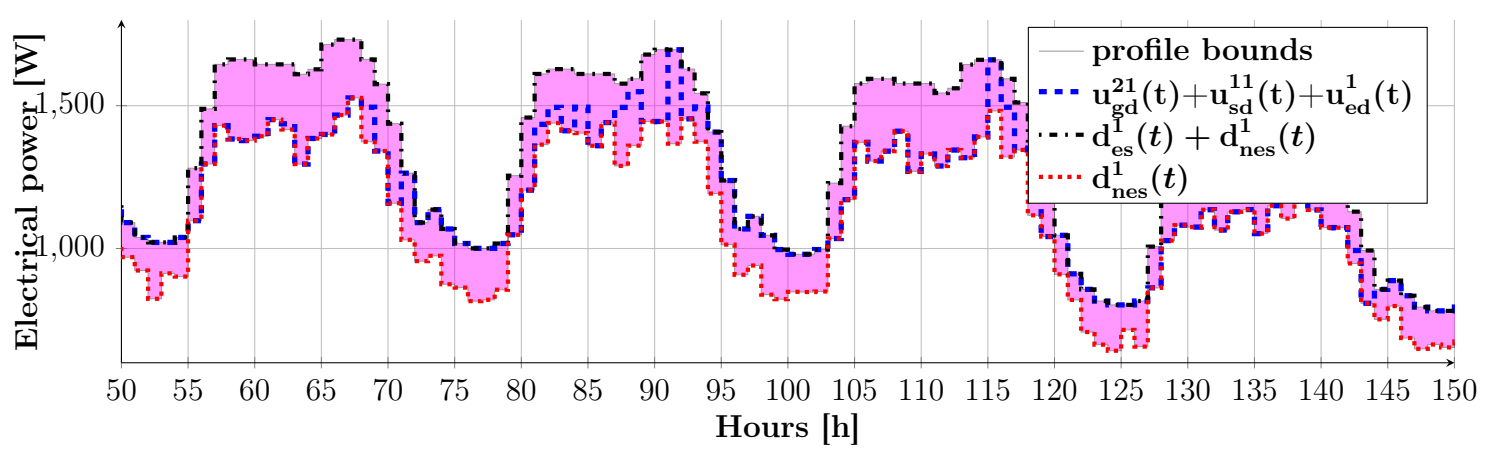

Figure 14: Electrical power transmitted by three sources to user 1.

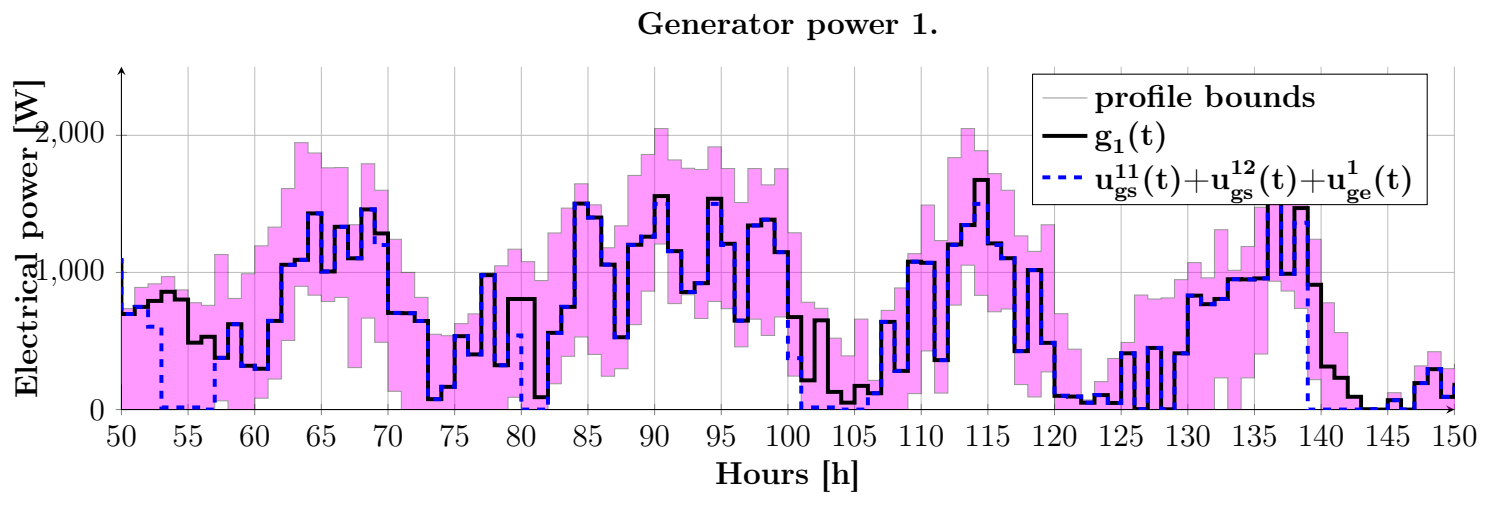

Figure 15: Electrical power consumed by user 1. 
Minimal battery capacity

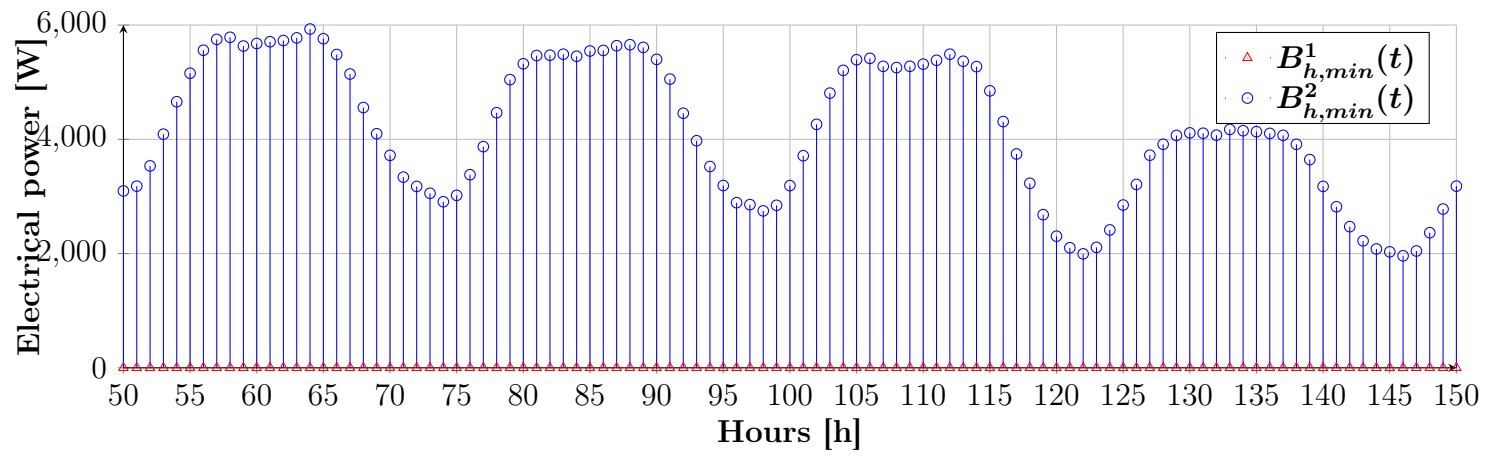

Figure 16: Minimal capacity of the battery storage units.

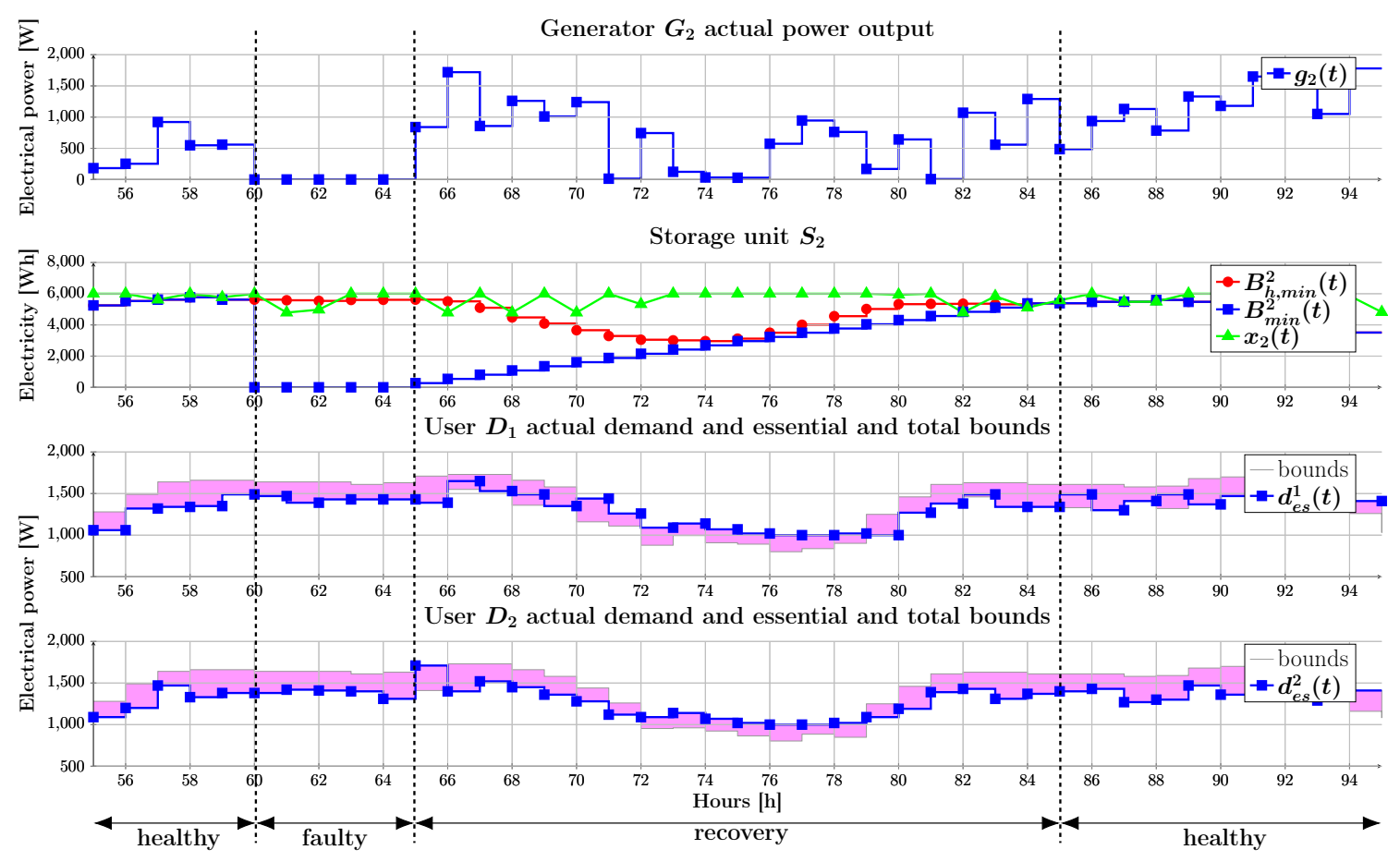

Figure 17: Microgrid system behavior under a fault event affecting generator $G_{2}$. 


\section{List of Tables}

1 General interconnection signals in a typical microgrid. . . . . . . . . . 41

2 Adjacency matrices specifying the links between the microgrid components. . . . . . . . . . . . . . . . . . . . 41

3 Numerical data for the microgrid components. . . . . . . . . . . . . . 42 


\begin{tabular}{|l|l|}
\hline \hline Notation & Description \\
\hline \hline$u_{g s}^{i j}(t)[W]$ & $\begin{array}{l}\text { electrical power transmitted by the generator } G_{i} \text { to the electrical } \\
\text { storage } S_{j} \text { at time step } t .\end{array}$ \\
$u_{g d}^{i k}(t)[W]$ & $\begin{array}{l}\text { electrical power transmitted by the generator } G_{i} \text { to the consumer } \\
D_{k} \text { at time step } t . \\
\text { electrical power transmitted by the generator } G_{i} \text { to the external } \\
\text { grid } E \text { at time step } t . \\
u_{g e}^{i}(t)[W]\end{array}$ \\
$u_{s d}^{j k}(t)[W]$ & $\begin{array}{l}\text { electrical power transmitted by the electrical storage } S_{j} \text { to the } \\
\text { consumer } D_{k} \text { at time step } t .\end{array}$ \\
$u_{s e}^{j}(t)[W]$ & $\begin{array}{l}\text { electrical power transmitted by the electrical storage } S_{j} \text { to the } \\
\text { external grid } E \text { at time step } t . \\
\text { electrical power transmitted by the external grid } E \text { to the consumer } \\
D_{k} \text { at time step } t .\end{array}$ \\
$u_{e d}^{k}(t)[W$
\end{tabular}

Table 1: General interconnection signals in a typical microgrid.

\begin{tabular}{|c|c|}
\hline Notation & Description \\
\hline$\overline{M_{g s}} \in\{0,1\}^{N_{g} \times N_{s}}$ & $\begin{array}{l}\text { characterizes the existence of a link from the generator } G_{i} \\
\text { to the electrical storage } S_{i} \text {. }\end{array}$ \\
\hline$M_{g d} \in\{0,1\}^{N_{g} \times N_{d}}$ & $\begin{array}{l}\text { characterizes the existence of a link from the generator } G_{i} \\
\text { to the consumer } D_{k} \text {. }\end{array}$ \\
\hline$M_{g e} \in\{0,1\}^{N_{g} \times 1}$ & $\begin{array}{l}\text { characterizes the existence of a link from the generator } G_{i} \\
\text { to the external grid } E \text {. }\end{array}$ \\
\hline$M_{s d} \in\{0,1\}^{N_{s} \times N_{d}}$ & $\begin{array}{l}\text { characterizes the existence of a link from the electrical } \\
\text { storage } S_{j} \text { to the consumer } D_{k} \text {. }\end{array}$ \\
\hline$M_{s e} \in\{0,1\}^{N_{s} \times 1}$ & $\begin{array}{l}\text { characterizes the existence of a link from the electrical } \\
\text { storage } S_{j} \text { to the external grid } E \text {. }\end{array}$ \\
\hline$M_{e d} \in\{0,1\}^{1 \times N_{d}}$ & $\begin{array}{l}\text { characterizes the existence of a link from the external grid } E \\
\text { to the consumer } D_{k} \text {. }\end{array}$ \\
\hline
\end{tabular}

Table 2: Adjacency matrices specifying the links between the microgrid components. 


\begin{tabular}{|c|c|c|c|c|c|c|c|c|}
\hline \multicolumn{9}{|c|}{ |Storage units parameters } \\
\hline \multicolumn{3}{|c|}{$\sigma_{1}=\sigma_{2}=13 \cdot 10^{-4} M=9 \cdot 10^{3}$} & \multicolumn{3}{|c|}{$B_{\min }^{1}=B_{\min }^{2}=12 \cdot 10^{2}[\mathrm{Wh}]$} & \multicolumn{3}{|c|}{$B_{\max }^{1}=B_{\max }^{2}=6 \cdot 10^{3}[W h]$} \\
\hline \multicolumn{3}{|c|}{$V_{\min }^{1}=V_{\min }^{2}=-1.5 \cdot 10^{3}[\mathrm{~W}]$} & \multicolumn{3}{|c|}{$V_{\max }^{1}=V_{\max }^{2}=1.5 \cdot 10^{3}[\mathrm{~W}]$} & & & \\
\hline \multicolumn{2}{|c|}{ Prediction horizon } & \multicolumn{7}{|c|}{ Control input constraints } \\
\hline \multicolumn{2}{|c|}{$N_{p}=7$} & \multicolumn{7}{|c|}{$\mathbf{u}=\left[\begin{array}{lllllllllllll}1.2 & 1.5 & 1.2 & 1.5 & 1.5 & 1.2 & 1.7 & 2 & 1.5 & 1.5 & 10^{-3} & 10^{-3}\end{array}\right]^{T} \cdot 10^{3}[\mathrm{~W}]$} \\
\hline \multicolumn{9}{|c|}{ Weight parameters in (13), (27) and (28) } \\
\hline$\gamma_{s, c}=0$ & $\gamma_{s, d}=0$ & \multicolumn{2}{|c|}{$\gamma_{s, s}=1$} & $\gamma_{b}=1$ & \multicolumn{2}{|c|}{$\gamma_{d}=0.5$} & $\gamma_{e}=1$ & $\gamma=1$ \\
\hline
\end{tabular}

Table 3: Numerical data for the microgrid components. 